\title{
CONTRIBUCIÓN AL CONOCIMIENTO CARIOLÓGICO DEL GÉNERO CENTAUREA L. (ASTERACEAE) EN LA PENÍNSULA IBÉRICA
}

\author{
Eusebio LÓPEZ1 y Juan Antonio DEVESA ${ }^{2 *}$
}

\begin{abstract}
${ }^{1}$ Departamento de Biología Vegetal, Ecología y Ciencias de la Tierra (Área de Botánica), Facultad de Ciencias, Universidad de Extremadura, Avenida de Elvas s/n, 06071-Badajoz, España.

${ }^{2}$ Departamento de Botánica, Ecología y Fisiología Vegetal, Facultad de Ciencias, Universidad de Córdoba, Campus de Rabanales, Edificio José Celestino Mutis, ctra. de Madrid km. 396, 14071-Córdoba, España

*Autor para correspondencia: bv1dealj@uco.es
\end{abstract}

Recibido el 17 de julio de 2008, aceptado para su publicación el 29 de septiembre de 2008 Publicado "on line" en octubre de 2008

RESUMEN. Contribución al conocimiento cariológico del género Centaurea L. (Asteraceae) en la Península Ibérica. Se da a conocer el número cromosómico de 22 táxones de Centaurea L. sect. Centaurea. En particular, se han estudiado 18 táxones del grupo de C. paniculata L., de los que $C$. paniculata subsp. exilis Arènes, C. paniculata subsp. geresensis Arènes, C. coutinhoi Franco, $C$. kheilii (Pau) Pau, C. limbata var. insularis Pau, C. castellanoides subsp. talaverae E. López \& Devesa, C. cordubensis Font Quer, C. bethurica E. López \& Devesa y C. schousboei Lange lo han sido por vez primera. Del grupo de $C$. alba L. se han estudiado 4 táxones, siendo nuevos los recuentos de $C$. costae Willk. var. costae y C. costae var. maluqueri Font Quer. En ambos grupos el número básico encontrado ha sido $x=9$. El número diploide $2 n=18$ es el más frecuente mientras que el nivel tetraploide $2 n=36(4 x)$ sólo se ha detectado en C. aristata Hoffmanns. \& Link, C. cordubensis Font Quer y C. schousboei Lange.

Palabras clave. Asteraceae, Centaurea, número cromosómico, Península Ibérica.

\begin{abstract}
A contribution to the karyological knowledge of the genus Centaurea L. (Asteraceae) in the Iberian Peninsula. A karyological study of 22 taxa of genus Centaurea L. sect. Centaurea is reported. Within C. paniculata L. group we have studied 18 taxa and the reports for some of them are given for the first time: C. paniculata subsp. exilis Arènes, C. paniculata subsp. geresensis Arènes, C. coutinhoi Franco, C. kheilii (Pau) Pau, C. limbata var. insularis Pau, C. castellanoides subsp. talaverae E. López \& Devesa, C. cordubensis Font Quer, C. bethurica E. López \& Devesa and C. schousboei Lange. Within $C$. alba L. group we have studied the chromosome number of 4 taxa, but only C. costae Willk. var. costae y C. costae var. maluqueri Font Quer are studied for the first time. In both groups we have found the basic number $x=9$. Diploid number $2 n=18$ is the most frequent whereas tetraploid level $2 n=36(4 x)$ has been found only in C. aristata Hoffmanns. \& Link, C. cordubensis Font Quer and C. schousboei Lange.
\end{abstract}

Key words. Asteraceae, Centaurea, chromosome number, Iberian Peninsula. 


\section{INTRODUCCIÓN}

Las Compuestas han sido objeto de múltiples estudios cariológicos, estimándose que a finales de la década de los 70 del pasado siglo se conocía ya el número cromosómico del 39\% (Solbrig, 1977) de las más de 23.000 especies que incluye la familia (Jeffrey, 2007), variando éste entre $2 n=4$ [en Haplopappus gracilis (Nutt) Gray y Brachyscome lineariloba (DC.) Druce; Tanaka, 1981, y Watanabe, Carter et al., 1976] y $2 n=180$ (Senecio roberti-friesii K. Afzel; Afzelius, 1924). Desde entonces, la información cariológica ha crecido considerablemente y, desde luego, en el género Centaurea en particular.

Los recuentos en Centaurea datan de la primera mitad del siglo XX (C. cyanus, $2 n$ =14; Poddubnaja-Arnoldi, 1927, y Morinaga et al., 1929), y hoy día se sabe que la variabilidad cromosómica en el género está comprendida entre $2 n=14$ (muchas especies) y $2 n=110$, número que presentan C. spruneri Boiss. \& Heldr. (Phitos, 1970) o C. kunkelii Garcia-Jacas (Garcia-Jacas, 1998). Las aportaciones que soportan este conocimiento han sido muy numerosas, entre ellas las de Mardsen-Jones \& Turrill, 1937; Roy, 1938; Guinochet, 1956 \& 1957; Guinochet \& Foissac, 1962; Runemark, 1967; Dipali, 1967; Tonian, 1968; Gardou, 1969; Phitos, 1970 y 1971; Bramwell et al., 1971 \& 1972; Fernandes \& Queirós, 1971; Gardou, 1972 a \& b; Queirós, 1973; Fernández Morales, 1974, sec. Agudo, 1981; Fernández Morales \& Gardou, 1975; Plitmann, 1976; Georgiadis \& Phitos, 1976; Matthäs, 1976; Damboldt \& Matthäs, 1975 \& 1979; Blanca, 1980, 1981 a, b, c; GarciaJacas \& Susanna, 1992; Garcia-Jacas et al., 1997 \& 1998 a y b; Ochsmann, 1999).

Especial mención merece el trabajo de Poddubnaja-Arnoldi (1931), quien estudia el número cromosómico de 14 especies, concluyendo que $x=9,10$ y 12 son los números básicos de los táxones del subgénero Cyanus, en el que centró su estudio. Destacar también el de Guinochet (1957), que estudió numerosas especies, representativas de 18 secciones del género Centaurea, concluyendo que: a) los números básicos en el género constituyen una serie disploide $(x=8,9,10,11,12,13$ y 15$)$, por translocaciones y pérdidas de porciones de cromosomas, y b) que hay un paralelismo entre las secciones establecidas sobre la base de características morfológicas (atendiendo sobre todo a las brácteas del involucro, el vilano y los caracteres polínicos) y los números cromosómicos hallados, pues las dotaciones cromosómicas de los representantes de las diferentes secciones estudiadas parecían ajustarse a un único número básico. En definitiva, para Guinochet (loc. cit.), los táxones más primitivos son aquellos con número básico elevado, capítulos grandes, con brácteas involucrales simples y más o menos enteras, con polen de tipo Serratula (más complejo) y hojas más o menos enteras y anchas, considerando como derivadas las secciones con números básicos más bajos, capítulos por lo general pequeños y con brácteas involucrales más o menos complejas, con polen de tipo Jacea (más simple) y hojas divididas. En un trabajo posterior, Guinochet \& Foissac (1962), estudian el cariotipo de 35 especies pertenecientes a 14 secciones, y establecen de nuevo relaciones entre los tipos polínicos definidos por Wagenitz (1955) y el tamaño de los cromosomas, observando que en las especies con polen de tipo Serratula (menos evolucionadas para Wagenitz) predominan los cromosomas pequeños, mientras que en las especies con polen de tipo Jacea (más evolucionados para Wagenitz) predominan lo cromosomas grandes.

En la actualidad, para el género Centaurea se conoce la serie de números 
básicos $x=7,8,9,10,11,12,13,14,15 \mathrm{y}$ 16 , y se acepta que esta serie disploide es el resultado de una reducción en el número cromosómico asociada a un incremento de la asimetría del cariotipo (Fernández Casas \& Fernández Morales, 1979; Fernández Casas \& Susanna, 1986; Siljak-Yakovlev, 1986), tendencia conocida también en otros géneros de la familia como, por ejemplo, Crepis L. y Leontodon L. (Stebbins, 1950 \& 1971). Los estudios con marcadores moleculares (Garcia-Jacas et al., 2001) han respaldado la fuerte correlación existente entre el número cromosómico y el tipo de polen, confirmándose así las ideas apuntadas por Guinochet (1957).

Garcia-Jacas et al. (1996), dentro la subtribu Centaureinae, sitúan en el número $2 n=24$ el límite cromosómico entre "lo primitivo" y "lo derivado", y consideran que los táxones con números cromosómicos superiores son los más primitivos, al tiempo que presentan polen de tipo Serratula o de tipo Centaurea centaurium, considerados también primitivos por Wagenitz (1955). A esta categoría pertenecen, por ejemplo, los táxones que tradicionalmente se reunían en la sección Centaurea, pero que integran un clado diferente al de las restantes especies de Centaurea (Garcia-Jacas et al., 2000), por lo que han sido segregadas en un género diferente: Rhaponticoides Vaill. (Gabrielian, 1995; Garcia-Jacas, Susanna et al., 2000; Greuter et al., 2001). Por el contrario, los táxones con número cromosómico $2 n=24$ ocupan una posición evolutiva intermedia, con granos de polen de los tipos Cyanus o Montana, como sucede con los representantes de Centaurea subgen. Cyanus $\mathrm{y}$, finalmente, aquellos táxones con números inferiores a $2 n=24$ integran grupos que se interpretan como más modernos, con polen de los tipos Centaurea scabiosa o Jacea. En el género, además, la poliploidía constituye un mecanismo de especiación o diferenciación frecuente, como extraordinariamente frecuentes -y a menudo viables- son también los cruces entre táxones, incluso con dotaciones cromosómicas de diferente número básico (Garcia-Jacas et al., 1996).

En el presente trabajo se ha abordado el estudio cariológico de diversas especies de la sección Centaurea -en su actual acepción- en la Península Ibérica, pertenecientes al grupo de C. paniculata $\mathrm{L}$. y al de C. alba L., que cuentan en el territorio con una abundante representación y alto grado de endemicidad. Dicha elección, aparentemente dispar, se fundamenta en el hecho de que los táxones de ambos grupos están íntimamente relacionados entre sí, y también con los de otras secciones (v.gr., Willkommia G. Blanca) del tradicional e inconsistente subgénero Acrolophus (Cass.) DC. (véase López \& Devesa, 2008 a), como han puesto de manifiesto diversas filogenias con marcadores moleculares (Font et al., 2002; Garcia-Jacas et al., 2006; SuárezSantiago et al., 2007). Los datos cariológicos aportados aquí derivan de una reciente revisión para ambos grupos en el marco del proyecto Flora Iberica (López, 2008) y que, a diferencia de los táxones de Centaurea gr. Willkommia, para los que se dispone de una revisión taxonómica relativamente reciente (Blanca, 1981a) y de abundante información cariológica (Blanca, 1980, 1981b \& c), se contaba en este caso solo con las revisiones de Arénes (1949 \& 1951) y la más reciente de Dostál (1976) a nivel europeo -al margen de los tratamientos parciales efectuados en algunas floras regionales-, y de algunas aportaciones cariológicas en algún caso no exentas de error por las conocidas dificultades en la identificación de los táxones.

Para los táxones del grupo de $C$. paniculata, los antecedentes indican que $2 n$ $=18$ es el número cromosómico dominante y $x=9$ el número básico, siendo fenómenos 


\begin{tabular}{|c|c|c|c|}
\hline TAXON & $2 n$ & ANTECEDENTES & ORIGEN DEL M ATERIAL \\
\hline C. schousboei Lange & 18 & Fernández Casas \& Gamarra, 1986 & $\begin{array}{c}\text { Entre Nocedo de Curueño y Tolibia de } \\
\text { Abajo (León, España) }\end{array}$ \\
\hline $\begin{array}{l}\text { C. spinabadia subsp. hanryi } \\
\text { (Jordan) Dostál }\end{array}$ & 18 & Agudo, 1981 & $\begin{array}{l}\text { Vilalleons - Vilatorta (Barcelona, } \\
\text { España) }\end{array}$ \\
\hline $\begin{array}{c}\text { C. spinabadia subsp. } \\
\text { shuttleworthii (Rouy) Dostál }\end{array}$ & 18 & Guinochet, 1957 & Trayas-Agay (Francia) \\
\hline \multirow[t]{5}{*}{$\begin{array}{c}\text { C. limbata Hoffmanns. \& } \\
\text { Link }\end{array}$} & 18 & Fernandes \& Queirós, 1971 & Barraçao y Valongo (Portugal) \\
\hline & 18 & Queirós, 1973 & Sezelhe y Penhas da Saúde (Portugal) \\
\hline & 18 & Valdés-Bermejo \& Gómez, 1976 & Finisterre (La Coruña, España) \\
\hline & 18 & Agudo, 1981 & Campelo (Lugo, España) \\
\hline & 18 & García Martínez, 2000 & Toques (La Coruña, España) \\
\hline $\begin{array}{l}\text { C. rothmalerana (Arènes) } \\
\text { Dostál }\end{array}$ & 18 & Caixinhas et al., 1991 & No indicado \\
\hline \multirow[t]{8}{*}{ C. paniculata L. } & 18 & Gardou, 1972b & $\begin{array}{c}\text { Alpes-Maritimes (Francia) y Alto } \\
\text { Adige (Italia) }\end{array}$ \\
\hline & $18(19)$ & $\begin{array}{c}\text { Guinochet, } 1956 \text { (sec. Fedorov, } \\
1974)\end{array}$ & \\
\hline & 18 & Van Loon \& De Jong, 1978 & Tesalónica (Grecia) \\
\hline & 18 & Natarajan, 1981 & Montpellier (Francia) \\
\hline & 18 & Natarajan, 1988 & Languedoc (Francia) \\
\hline & 18 & Ochsmann, 1999 & Bragniols, Le Val (Francia) \\
\hline & 18 & Kiehn et al., 2000 & Austria \\
\hline & 18 & Guinochet, 1957 & Brignais-Givors (Francia) \\
\hline $\begin{array}{c}\text { C. paniculata subsp. rigidula } \\
\text { (Jord.) Dostál }\end{array}$ & 18 & Guinochet, 1957 & Cap d'Antibes (Francia) \\
\hline $\begin{array}{l}\text { C. paniculata subsp. } \\
\text { esterellensis (Burnat) Dostál }\end{array}$ & 18 & Guinochet, 1957 & Agay (Francia) \\
\hline $\begin{array}{c}\text { C. paniculata subsp. } \\
\text { castellana (Boiss. \& Reut.) } \\
\text { Dostál }\end{array}$ & 18 & $\begin{array}{l}\text { Fernández Morales, } 1974 \text { (sec. Agudo, } \\
1981 \text { ) }\end{array}$ & Jaén, Granada \\
\hline & 18 & Agudo, 1981 & $\begin{array}{l}\text { Albacete-Albadalejo (Albacete, } \\
\text { España) }\end{array}$ \\
\hline & 36 & Agudo, 1981 & Alpedrete (Madrid, España) \\
\hline & 18 & Fernández Casas \& Gamarra, 1986 & $\begin{array}{l}\text { Entre Medinaceli y Almazán (Soria, } \\
\text { España) }\end{array}$ \\
\hline \multirow[t]{3}{*}{ C. leucophaea Jord. } & 18 & Guinochet, 1957 & Thorenç (Francia) \\
\hline & 18 & Delay, 1969 & Digne (Francia) \\
\hline & 18 & Ochsmann, 1999 & Liguria (Italia) \\
\hline $\begin{array}{l}\text { C. leucophaea subsp. reuteri } \\
\text { (Rchb. fil.) Dostál }\end{array}$ & 18 & Guinochet, 1957 & Escragnolles (Francia) \\
\hline C. castellanoides Talavera & 18 & Aparicio, 1993 & Sierra de Grazalema (Cádiz, España) \\
\hline
\end{tabular}

Tabla 1. Números cromosómicos indicados en los táxones del grupo de Centaurea paniculata. 


\begin{tabular}{|c|c|c|c|}
\hline TAXON & $2 n$ & AUTOR, AÑ̃ & ORIGEN DEL MATERIAL \\
\hline \multirow[t]{6}{*}{ C. alba $\mathrm{L}$. } & 18 & Gardou, 1972a & Somosierra (Madrid, España) \\
\hline & 18 & Matthäs, 1976 & $\begin{array}{c}\text { Sierra de Gredos (Madrid, } \\
\text { España) }\end{array}$ \\
\hline & 18 & Van Loon \& Kieft, 1980 & Visegrad (Bosnia-Herzegovina) \\
\hline & 36 & Kuzmanov et al., 1986 & Bulgaria \\
\hline & 18 & Hellwig, 1994 & España, varias localidades \\
\hline & 18 & Ochsmann, 2000 & No Indicado \\
\hline $\begin{array}{c}\text { C. alba subsp. tartesiana } \\
\text { Talavera }\end{array}$ & 18 & Pastor et al., 1990 & La Umbría (Huelva, España) \\
\hline \multirow[t]{2}{*}{$\begin{array}{l}\text { C. alba subsp. macrocephala } \\
\text { (Pau) Talavera }\end{array}$} & 18 & Agudo, 1981 & $\begin{array}{l}\text { Navalperal de Pinares (Ávila, } \\
\text { España) }\end{array}$ \\
\hline & 18 & Agudo, 1981 & Navacerrada (Madrid, España) \\
\hline \multirow[t]{4}{*}{$\begin{array}{l}\text { C. alba subsp. deusta (Ten.) } \\
\text { Nyman }\end{array}$} & 18 & Damboldt et al., 1973 & Mattinata (Italia) \\
\hline & $18+1 \mathrm{~B}$ & Matthäs, 1976 & Plagia, Campania (Italia) \\
\hline & 18 & Šljak -Yakovlev, 1977 & Dubrovnik (Croacia) \\
\hline & 18 & Constantinidis \& Kamari, 1995 & Sterea Ellas (Grecia) \\
\hline $\begin{array}{c}\text { C. alba subsp. heldreichii } \\
\text { Halacsy }\end{array}$ & 18 & Phitos \& Damboldt, 1971 & Etolia-Acarnania (Grecia) \\
\hline $\begin{array}{l}\text { C. alba subsp. diomedea } \\
\text { Gasp. }\end{array}$ & 18 & Speta, 1971 & Tremiti, Isla San Incola (Italia) \\
\hline \multirow[t]{5}{*}{$\begin{array}{l}\text { C. alba subsp. subcialiaris } \\
\text { (Boiss. \& Heldr.) Dostál }\end{array}$} & $18+2 B$ & Phitos \& Damboldt, 1971 & Cefalonia (Grecia) \\
\hline & 36 & Phitos \& Damboldt, 1971 & Cefalonia (Grecia) \\
\hline & $36+4 B$ & Phitos \& Damboldt, 1971 & Cefalonia (Grecia) \\
\hline & 36 & Matthäs, 1976 & Cefalonia (Grecia) \\
\hline & 18 & Matthäs, 1976 & Lefkas (Grecia) \\
\hline C. splendens $\mathrm{L}$. & 20 & Nilsson \& Lassen, 1971 & Novi Vimodolski (Croacia) \\
\hline $\begin{array}{l}\text { C. deusta subsp. divaricata } \\
\text { Matthäs \& Pignatti }\end{array}$ & 18 & Brullo et al., 1991 & Torre d'In serraglie (Italia) \\
\hline \multirow[t]{2}{*}{ C. huljakii J. Wagner } & 18 & Damboldt \& Melzheimer, 1974 & Peninsula de Athos (Grecia) \\
\hline & 36 & Matthäs, 1976 & Peninsula de Athos (Grecia) \\
\hline C. ferulacea Martelli & 18 & Arrigoni \& Mori, 1971 & Cerdeña (Italia) \\
\hline \multirow[t]{2}{*}{ C. musarum Boiss. \& Orph. } & 18 & Kamari, 1996 & No Indicado \\
\hline & 18 & Constantinidis \& Kamari, 2000 & Nomos Viotias (Grecia) \\
\hline C. aziziana Rech. f. & 18 & Garcia-Jacas et al., 1998b & Golfa (Irán) \\
\hline
\end{tabular}

Tabla 2. Números cromosómicos indicados en los táxones del grupo de Centaurea alba. 
de poliploidía los que explican el recuento efectuado en algunas de las especies (tab. 1), y lo mismo sucede en el grupo de C. alba (tab. 2). El número $2 n=20$ encontrado por Nilsson \& Lassen (1971) en material croata de C. splendens L. se explica, probablemente, por la presencia de cromosomas supernumerarios, un fenómeno también común en el grupo y ya apuntado en algunos táxones [v.gr., C. alba subsp. subciliaris (Boiss. \& Heldr.) Dostál; véase tabla 2].

En conjunto, se aporta información sobre 18 táxones del grupo de $C$. paniculata y 4 del grupo de C. alba, muchos de los cuales (12) han sido estudiados por primera vez desde el punto de vista cariológico.

\section{MATERIAL Y MÉTODOS}

El estudio de los cromosomas se ha efectuado en divisiones mitóticas de células meristemáticas de raíces, obtenidas a partir de plántulas crecidas de aquenios recolectados en el campo.

Como antimitótico se utilizó 8hidroxiquinoleina 0,002 M (Tjio \& Levan, 1950), actuando durante 4 horas a temperatura ambiente, y como fijador una mezcla de alcohol absoluto y acetato férrico (3:1) durante al menos 24 h, conservándose a continuación las muestras en viales con alcohol al 70\%. Para la tinción se utilizó carmín alcohólico-acético (Snow, 1963), siendo suficientes 24-48 horas para alcanzar la tinción adecuada en la gran mayoría de los casos. El montaje de las muestras se efectuaba en portaobjetos con una gota de ácido acético al $45 \%$, procediéndose al aplastamiento de las mismas tras taparlas con un cubreobjetos, quedando así listas para su observación al microscopio.

Para la descripción de los cromosomas se ha utilizado la terminología de Levan et al. (1964: m, metacéntricos; sm, submetacéntricos; st, subtelocéntricos y t, telocéntricos), y la asimetría cariotípica indicada se ajusta a la clasificación propuesta por Stebbins (1971) y modificada por Dvorak et al. (1979), donde A1 representaría el mayor grado de simetría y $\mathrm{C} 4$ el mayor grado de asimetría. El tamaño de los cromosomas se indica mediante tres valores expresados en micras: el primero referido a la longitud del cromosoma más grande, el segundo (entre paréntesis) a la longitud media y su desviación típica, y el tercero al tamaño del cromosoma más pequeño.

Por último, indicar que para cada población se han estudiado varias placas metafásicas y que, tanto para la asimetría como para el tamaño de los cromosomas, se dan los valores medios de los cariotipos estudiados para cada población.

\section{RESULTADOS}

CENTAUREA GR. C. PANICULATA L.

1. C. paniculata L., Sp. Pl.: 912 (1753) subsp. paniculata

Material estudiado. ESPAÑA. Gerona. Carretera de San Martín de Seserres al monasterio de la Mare de Deu del Mont, 22-VII-2004, J. López \& E. López (UNEX 34481).

Se ha encontrado el número cromosómico $2 n=18$ (fig. 1, A-B), que coincide con el señalado en material de diversos países europeos (tab. 1), si bien los recuentos de Kiehn et al. (2000) en plantas de Austria hay que referirlos casi con toda probabilidad a $C$. maculosa o $C$. rhenana. La fórmula cromosómica es $8 \mathrm{~m}+1 \mathrm{sm}$, la asimetría cariotípica de tipo A1 y el tamaño de los cromosomas viene definido por los valores 3,1 $\mathrm{mm}-(2,51 \mathrm{~mm} \pm 0,42)-1,7 \mathrm{~mm}$.

2. C. paniculata subsp. leucophaea (Jord.) Arcang., Comp. Fl. Ital.: 392 (1882)

C. leucophaea Jord., Observ. Pl. Nouv. 5: 64 (1847)

Material estudiado. ESPAÑA. Gerona. 

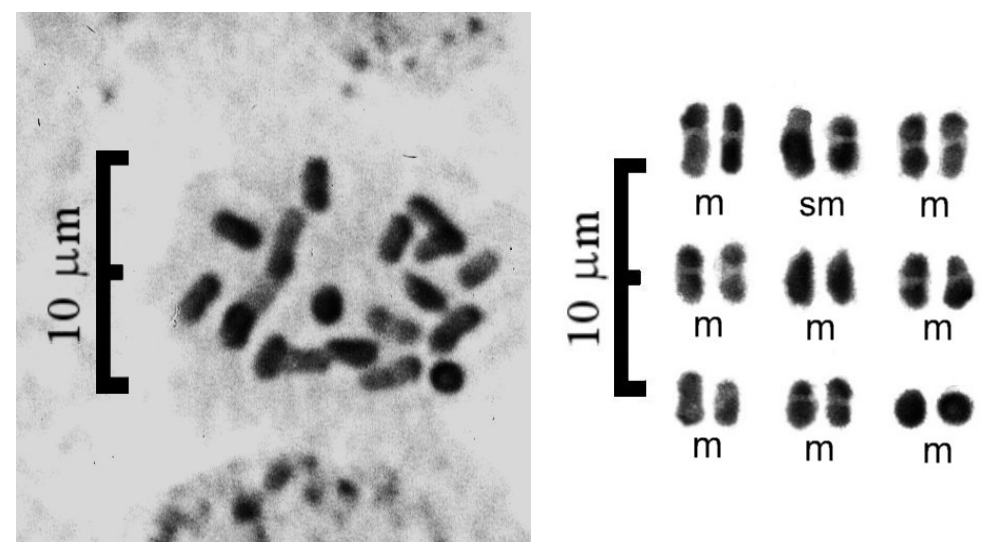

A

B
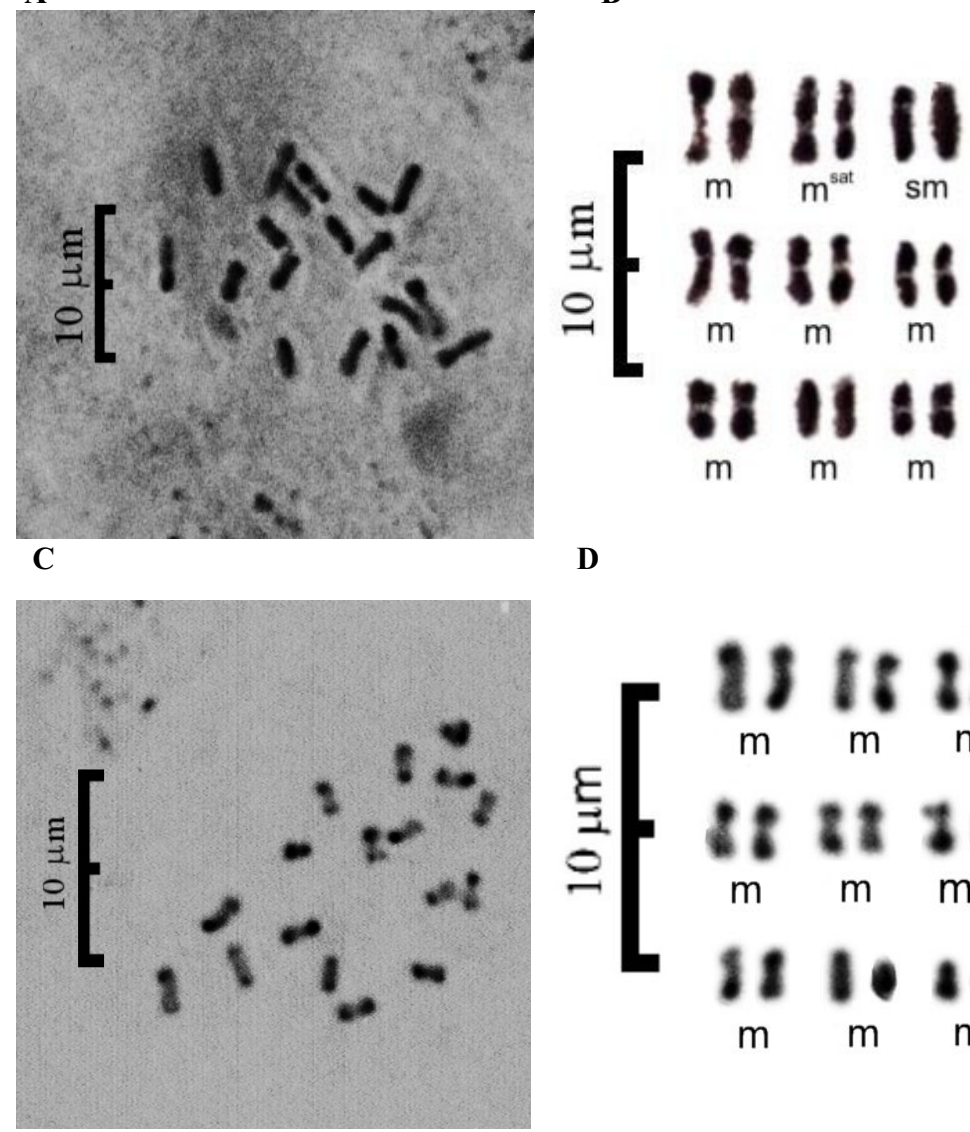

D

$\mathbf{E}$

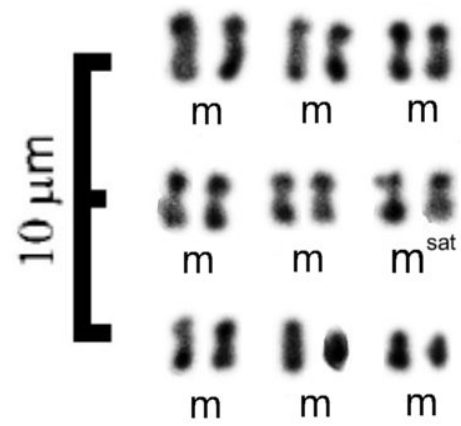

F

Figura 1. Metafases somáticas y cariotipos de: A-B, C. paniculata subsp. paniculata (Gerona, UNEX 34481); C-D, C. paniculata subsp. leucophaea (Gerona, COFC 30732); E-F, C. paniculata subsp. exilis. (Beira Alta, COFC 30696). 
Romanyá de la Selva, 23-VII-2004, J. López \& E. López (COFC 30732).

En plantas de la única población estudiada se ha encontrado el número cromosómico $2 n=$ 18 (fig. 1, C-D), que no discrepa del señalado previamente en plantas del sur de Francia y el noroeste de Italia (tab. 1). La fórmula cromosómica es $7 \mathrm{~m}+1 \mathrm{~m}^{\text {sat }}+1 \mathrm{sm}$, la asimetría cariotípica de tipo A1 y el tamaño de los cromosomas viene definido por los valores 4,2 $\mathrm{mm}-(3,16 \mathrm{~mm} \pm 0,51)-2,4 \mathrm{~mm}$.

3. C. paniculata subsp. exilis Arènes in Agron. Lusit. 11(1): 20 (1949)

Material estudiado. PORTUGAL. Beira Alta. Oliveira do Conde, 16-VII-2003, Devesa \& E. López (COFC 30696). Beira Baixa. Alcaide, 16-VII-2003, Devesa \& E. López (COFC 30692).

En las dos poblaciones estudiadas se ha encontrado el número cromosómico $2 n=18$ (fig. 1, E-F), no conociéndose recuento previo para este endemismo del CW de la Península Ibérica. La fórmula cromosómica encontrada en las plantas de Oliveira do Conde es $8 \mathrm{~m}+1 \mathrm{~m}^{\text {sat }}$, la asimetría cromosómica de tipo A1 y el tamaño de sus cromosomas de $2,6 \mathrm{~mm}-(2,01 \mathrm{~mm} \pm 0,31)$ - $1,4 \mathrm{~mm}$.

4. C. paniculata subsp. geresensis Arènes in Agron. Lusit. 11(1): 22 (1949)

Material estudiado. PORTUGAL. Minho. Carretera de Arcos de Valdevez a Marçao, 22VI-2004, J. López \& E. López (COFC 30693); Vilar de Veiga, 22-VI-2004, J. López \& E. López (UNEX 34468).

El número cromosómico $2 n=18$ (fig. 2, AB) encontrado en ambas poblaciones es, probablemente, el primer recuento para este endemismo del NW de la Península Ibérica (Sierra de Peneda-Gerês, y áreas adyacentes). La fórmula cromosómica encontrada en plantas de la población situada entre Arcos de Valdevez y Marçao es $8 \mathrm{~m}+1 \mathrm{sm}$, la asimetría de tipo A1 y el tamaño cromosómico de $3,2 \mathrm{~mm}-(2,58 \mathrm{~mm} \pm$ $0,32)-2,0 \mathrm{~mm}$.

5. C. paniculata subsp. rothmalerana Arènes in Agron. Lusit. 11(1): 24 (1949)

Material estudiado. PORTUGAL. Beira

Alta. Entre Covilha y Penhas de Saúde, 16-VII-
2003, Devesa \& E. López (UNEX 34469); entre Penhas da Saude y Manteigas, 16-VII-2003, Devesa \& E. López (COFC 30717).

En las dos poblaciones estudiadas se ha encontrado el mismo número, $2 n=18$ (fig. 2, CD), así como fórmulas $\left[7 \mathrm{~m}+1 \mathrm{~m}^{\text {sat }}+1 \mathrm{sm}\right.$, UNEX 34469 , y $6 \mathrm{~m}+2 \mathrm{sm}+1 \mathrm{sm}^{\text {sat }}$, COFC 30717] y tamaño de los cromosomas $[4,4 \mathrm{~mm}-(3,11 \mathrm{~mm}$ $\pm 0,58)-2,3 \mathrm{~mm}, \mathrm{UNEX} 34469, \mathrm{y} 4,3 \mathrm{~mm}-(3,20$ $\mathrm{mm} \pm 0,60)-2,6 \mathrm{~mm}$, COFC 30717] similares, $\mathrm{y}$ asimetría de tipo A1. Para este taxon, endémico de la Sierra de la Estrella (Portugal), se conocía ya el recuento de Caixinhas et al. (1991), y tal vez haya que referir también a él el observado por Queirós (1973) en plantas de la Sierra de la Estrella (Portugal) y que identifica como $C$. paniculata subsp. limbata.

6. C. coutinhoi Franco in Nova Fl. Portugal 2: 477, 572 (1984)

Material estudiado. ESPAÑA. Cáceres. Aceña de La Borrega, 22-V-2003, E. López \& Valtueña (UNEX 34475). PORTUGAL. Alto Alentejo. Castelo de Vide, 16-VII-2003, Devesa \& E. López (COFC 30744); Marvao, 4-VI-2005, Valtueña (COFC 30684).

En las tres poblaciones estudiadas se ha encontrado el mismo número, $2 n=18$ (fig. 2 , EF), y fórmulas cromosómicas similares: $6 \mathrm{~m}+3 \mathrm{sm}$ (UNEX 34475), 8m + 1sm (COFC 30744) y $7 \mathrm{~m}$ $+2 \mathrm{sm}$ (COFC 30684). De igual forma, en los tres casos los cariotipos presentan una asimetría de tipo A1 y el tamaño de los cromosomas es muy parecido: $3,1 \mathrm{~mm}-(2,57 \mathrm{~mm} \pm 0,44)-1,9 \mathrm{~mm}$ (UNEX 34475); 2,9 mm - $(2,23 \mathrm{~mm} \pm 0,40)-1,9$ $\mathrm{mm}(\mathrm{COFC} 30744)$ y $3,1 \mathrm{~mm}-(2,49 \mathrm{~mm} \pm 0,40)$ $-1,9 \mathrm{~mm}$ (COFC 30684). Se trata de los primeros recuentos efectuados para este endemismo hispano-lusitano del CW de la Península Ibérica.

7. C. kheilii (Pau) Pau in Bol. Soc. Aragonesa Ci. Nat. 1: 49 (1902)

C. coerulescens var. kheili Pau in Actas Soc. Esp. Hist. Nat. 26: 200 (1897)

Material estudiado. ESPAÑA. Cáceres. Hervás, 13-VII-2004, Devesa \& E. López (COFC 30707).

En las plantas de la única población estudiada se ha encontrado el número cromosómico $2 n=18$ (fig. 3, A-B). La fórmula 

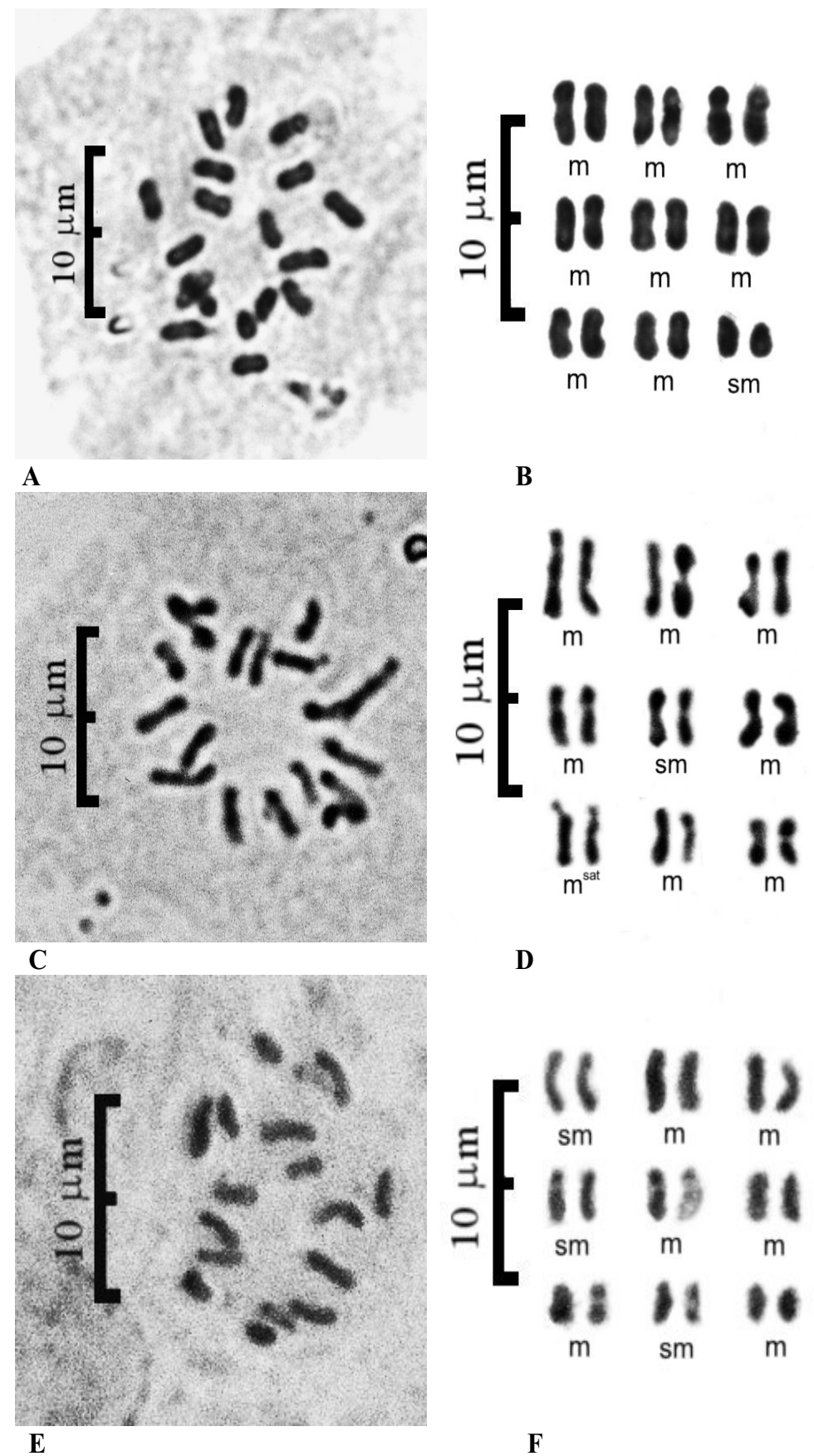

Figura 2. Metafases somáticas y cariotipos de: A-B, C. paniculata subsp. geresensis (Minho, COFC 30693); C-D, C. paniculata subsp. rothmalerana (Beira Alta, UNEX 34469); E-F, C. coutinhoi. (Alto Alentejo, COFC 30744). 
cromosómica es $6 \mathrm{~m}+3 \mathrm{sm}$, la asimetría cariotípica de tipo A1 y el tamaño de los cromosomas viene definido por los valores 2,8 $\mathrm{mm}-(2,10 \mathrm{~mm} \pm 0,26)-1,9 \mathrm{~mm}$. Se trata del primer recuento efectuado para este endemismo del W del Sistema Central de la Península Ibérica.

\section{C. hanryi Jord., Observ. Pl. Nouv. 5: 70 (1847)} Material estudiado. ESPAÑA. Barcelona. Barcelona, Monte Tibidabo, 17-VI-2005, E. López (COFC 30729); entre Matadepera y La Barata, 17-VI-2005, E. López (UNEX 34480).

En las plantas procedentes del Monte Tibidabo se ha encontrado el número cromosómico $2 n=18$ (fig. 3, C-D), coincidente con el señalado por Agudo (1981) en plantas de una localidad cercana, pero en las recolectadas entre Matadepera y La Barata (UNEX 34480) se detectó en algunas placas metafásicas la presencia de 1 cromosoma accesorio $(2 n=18+1 \mathrm{~B})$. Las fórmulas cromosómicas $6 \mathrm{~m}+3 \mathrm{sm}$ (COFC 30729) y $7 \mathrm{~m}+1 \mathrm{~m}^{\text {sat }}+1 \mathrm{sm}$ (UNEX 34480) son similares, e igual sucede con el tamaño de los cromosomas, que viene definido por los valores $3,7 \mathrm{~mm}$ - $(3,04$ $\mathrm{mm} \pm 0,48)-2,3 \mathrm{~mm}($ COFC 30729$)$ y $2,9 \mathrm{~mm}-$ $(2,39 \mathrm{~mm} \pm 0,36)-1,7 \mathrm{~mm}$ (UNEX 34480), en ambos casos con asimetría de tipo A1.

9. C. limbata Hoffmanns. \& Link, Fl. Portug. 2: 221 (1820-1828) var. limbata

Material estudiado. ESPAÑA. La Coruña. Cabo Finesterre, 23-VI-2004, J. López \& E. López (UNEX 34471).

En la única población estudiada de este endemismo hispano-lusitano del NW de la Península Ibérica se ha encontrado el número cromosómico $2 n=18$ (fig. 3, E-F). La fórmula cromosómica es $8 \mathrm{~m}+1 \mathrm{~m}^{\text {sat }}$, la asimetría de tipo A1 y el tamaño de los cromosomas viene definido por los valores $2,2 \mathrm{~mm}-(1,79 \mathrm{~mm} \pm 0,27)-1,2$ $\mathrm{mm}$.

El recuento efectuado coincide (tab. 1) con los de Fernandes \& Queirós (1971) y Queirós (1973) en plantas de poblaciones portuguesas; con el de García Martínez (2000), en material procedente de Toques (La Coruña), y con el de Valdés-Bermejo \& Gómez (1976) en plantas también de Finisterre en las que, no obstante, señalaron la presencia de 1 par de cromosomas satelizados. Agudo (1981) señala en plantas de la provincia de Lugo un tamaño de los cromosomas algo mayor, entre 2 y $3,5 \mathrm{~mm}$, ajustándose el material que estudió a la fórmula $2 \mathrm{~m}+7 \mathrm{sm}$.

10. C. limbata var. insularis $P a u$ in Bol. Soc. Aragonesa Ci. Nat. 1: 49 (1902)

Material estudiado. ESPAÑA. Pontevedra. Islas Cíes, isla de Montefaro, 26-VII-2006, J. López \& E. López (UNEX 34490).

En plantas de esta población se ha encontrado el número cromosómico $2 n=18$, tratándose al parecer del primer recuento efectuado en este taxon endémico de las Islas Cíes (Pontevedra).

11. C. langeana Willk. in Willk. \& Lange, Prodr. Fl. Hispan. 2: 157 (1870) subsp. langeana Material estudiado. ESPAÑA. Salamanca. La Fregeneda, 30-VI-2003, Ortega \& E. López (COFC 30713); Masueco, carretera a Pereña, 1VII-2003, Ortega \& E. López (COFC 30709).

Se ha encontrado el número cromosómico $2 n=18$ (fig. 4, A-B), coincidente con el hallado previamente por Fernández Casas \& Gamarra (1986) en plantas procedentes de la provincia de León (sub C. schousboei Lange, tab. 1) y, probablemente, también haya que referir a este taxon el recuento efectuado por estos autores en una población procedente de Soria e identificada como C. paniculata subsp. castellana, aunque en este caso no ha podido estudiarse el testigo.

En las dos poblaciones la asimetría es de tipo A 1 y las fórmulas cromosómicas muy parecidas, $9 \mathrm{~m}$ (COFC 30713) y $8 \mathrm{~m}+1 \mathrm{sm}$ (COFC $30709)$, al igual que lo es el tamaño de los cromosomas: $2,7 \mathrm{~mm}-(2,10 \mathrm{~mm} \pm 0,39)-1,5$ $\mathrm{mm}(\mathrm{COFC} 30713)$ y $2,5 \mathrm{~mm}-\left(1,88^{\prime} \mathrm{mm} \pm 0,36\right)$ $-1,3 \mathrm{~mm}$ (COFC 30709).

12. C. aristata Hoffmanns. \& Link, Fl. Portug. 2: 226 (1820-1828)

$=$ C. castellana Boiss. \& Reut. in Boiss., Diagn. Pl. Orient. ser. 1, 6: 129 (1842)

Material estudiado. ESPAÑA. Madrid. Pelayos de la Presa, en dirección a Robledo de Chavela, 7-VII-2005, E. López (COFC 30739); San Martín de Valdeiglesias, carretera a la ermita Virgen de la Nueva, 7-VII-2005, E. López (COFC 30686). 


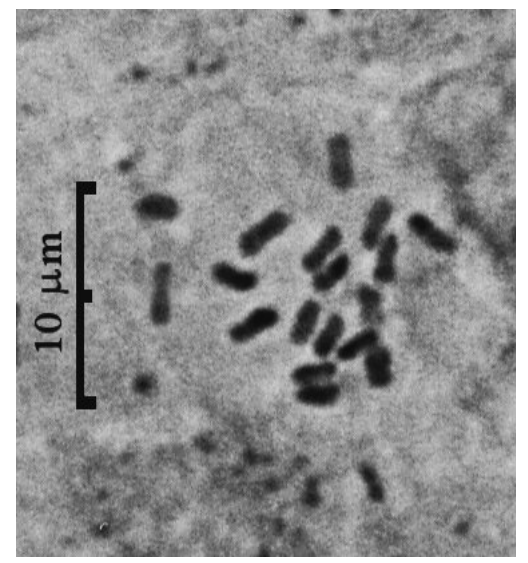

A
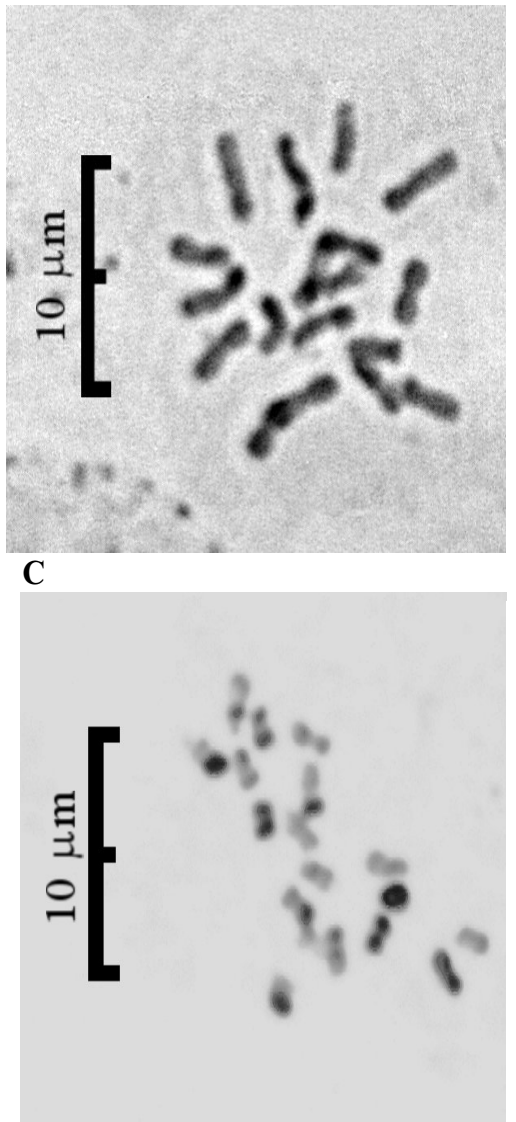

$\mathbf{E}$

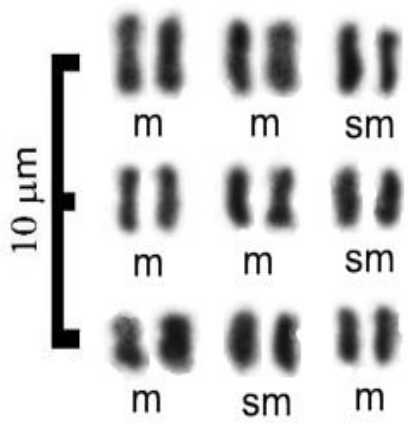

B

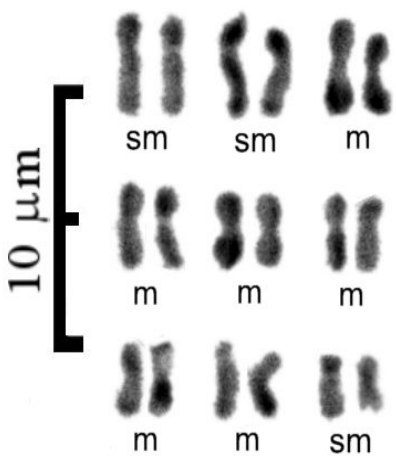

D

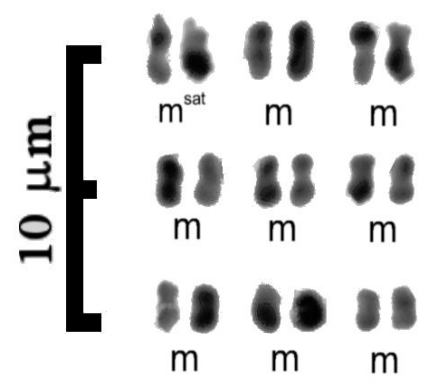

F

Figura 3. Metafases somáticas y cariotipos de: A-B, C. kheilii (Cáceres, COFC 30707); C-D, C. hanryi (Barcelona, COFC 30729); E-F, C. limbata var. limbata (La Coruña, UNEX 34471). 
En plantas de las dos poblaciones estudiadas se ha encontrado el número $2 n=36$ (fig. $4, \mathrm{C}-\mathrm{D}$ ), que pone de manifiesto el origen poliploide ( $4 x$, $x=9$ ) de este endemismo de la Península Ibérica. En ambos casos los cariotipos presentan una asimetría de tipo A1, siendo las fórmulas cromosómicas $15 \mathrm{~m}+1 \mathrm{~m}^{\text {sat }}+2 \mathrm{sm}^{\text {sat }}(\mathrm{COFC}$ $30739)$ y $14 \mathrm{~m}+4 \mathrm{sm}$ (COFC 30686$)$, y el tamaño de los cromosomas muy similar: $3,0 \mathrm{~mm}-(2,61$ $\mathrm{mm} \pm 0,39)-2,2 \mathrm{~mm}($ COFC 30739$)$ y $3,9 \mathrm{~mm}-$ $(2,87 \mathrm{~mm} \pm 0,45)-2,8 \mathrm{~mm}(\mathrm{COFC} 30686)$.

Los recuentos efectuados coinciden (tab. 1) con el número indicado por Agudo (1981, sub $C$. castellana) en plantas de una población de Alpedrete (Madrid), pero no así con el que halló en plantas procedentes de Albacete $(2 n=18)$, aunque probablemente éstas no se corresponden con este taxon, sino que deban identificarse con C. castellanoides, lo que probablemente le ocurra también al material testigo de los recuentos efectuados por Fernández Morales (1974, sec. Agudo, 1981). De igual manera, es probable que el conteo efectuado por Fernández Casas \& Gamarra (1986, sub C. castellana) deba referirse a C. castellanoides subsp. talaverae (vide López \& Devesa, 2008b).

13. C. castellanoides Talavera in Lagascalia 12: 271 (1984) subsp. castellanoides

Aunque el taxon no ha podido ser estudiado, probablemente hay que referir a él el número cromosómico $2 n=18$ indicado por Agudo (1981) en plantas procedentes de Jaén e identificadas como C. paniculata subsp. castellana, y por Fernández Morales (1974, sec. Agudo, 1981) en material con igual identificación y procedente de Jaén y Granada.

14. C. castellanoides subsp. arundana E. López \& Devesa in Acta Bot. Malacitana 33: 60 (2008)

Material estudiado. ESPAÑA. Málaga. Parque Natural Sierra de las Nieves, 10-VII-2004, E. López \& Valtueña (UNEX 34477).

En la población estudiada de este endemismo del S de España (López \& Devesa, 2008 b) se ha encontrado el número cromosómico $2 n=18$ (fig. 4, E-F), que coincide con el obtenido por Aparicio (1993) en plantas de la Sierra de Grazalema (Cádiz, sub C. castellanoides). La fórmula cromosómica hallada es $7 \mathrm{~m}+2 \mathrm{sm}$, la asimetría cariotípica de tipo A1 y el tamaño de los cromosomas viene definido por los valores $3,8 \mathrm{~mm}-(3,24 \mathrm{~mm} \pm 0,32)-2,8 \mathrm{~mm}$.

15. C. castellanoides subsp. talaverae E. López \& J.A. Devesa in Acta Bot. Malacitana 33: 60 (2008)

Material estudiado. ESPAÑA. Albacete. carretera entre La Hoz y Alcaraz, 30-VII-2004, E. López (UNEX 35212). Toledo. Noblejas, carretera en dirección a Dos Barrios, 7-VII-2005, E. López (UNEX 34478).

Se han estudiado plantas procedentes de dos poblaciones, obteniéndose en ambos casos el mismo número cromosómico, $2 n=18$ (fig. 5 , AB). Las fórmulas cromosómicas son $7 \mathrm{~m}+2 \mathrm{sm}$ (UNEX 35212) y $4 \mathrm{~m}+4 \mathrm{sm}+1 \mathrm{sm}^{\text {sat }}$ (UNEX 34478), la asimetría de tipo A1 y el tamaño de los cromosomas varía entre $3,2 \mathrm{~mm}-(2,71 \mathrm{~mm}$ $\pm 0,28)-2,4 \mathrm{~mm}($ UNEX 35212$)$ y $5,2 \mathrm{~mm}-(4,10$ $\mathrm{mm} \pm 0,60)-3,4 \mathrm{~mm}$ (UNEX 34478). Se trata de los primeros recuentos efectuados para este endemismo del SE y CE de la Península Ibérica (López \& Devesa, 2008b).

16. C. cordubensis Font Quer in Collect. Bot. (Barcelona) 1(3): 310 (1947)

Material estudiado. ESPAÑA. Badajoz. Fuente de Cantos, carretera a Llerena, 22-VII2005, E. López (UNEX 34473). Huelva. E1 Marquesado, 14-VI-2006, Devesa et al. (UNEX 35206).

En plantas de las dos poblaciones estudiadas se ha encontrado $2 n=36$ (fig. 5, C-D), número que pone de manifiesto el origen poliploide $(4 x$, $x=9$ ) de este taxon endémico del CS y SW de la Península Ibérica (López \& Devesa, 2008b) que, al parecer, se estudia por vez primera. En ambos casos los cariotipos presentan una asimetría de tipo A1 y se ajustan a las fórmulas $16 \mathrm{~m}+2 \mathrm{sm}$ (UNEX 34473 ) y $14 \mathrm{~m}+4 \mathrm{sm}+1 \mathrm{sm}^{\text {sat }}$ (UNEX 35206), variando los cromosomas entre $3,2 \mathrm{~mm}$ $-(2,64 \mathrm{~mm} \pm 0,38)-2,2 \mathrm{~mm}($ UNEX 34473$) \mathrm{y}$ $3,2 \mathrm{~mm}-(2,40 \mathrm{~mm} \pm 0,40)-2,0 \mathrm{~mm}$ (UNEX 35206).

17. C. bethurica E. López \& J.A. Devesa in Anales Jard. Bot. Madrid 65: 337 (2008) Material estudiado. ESPAÑA. Badajoz. 

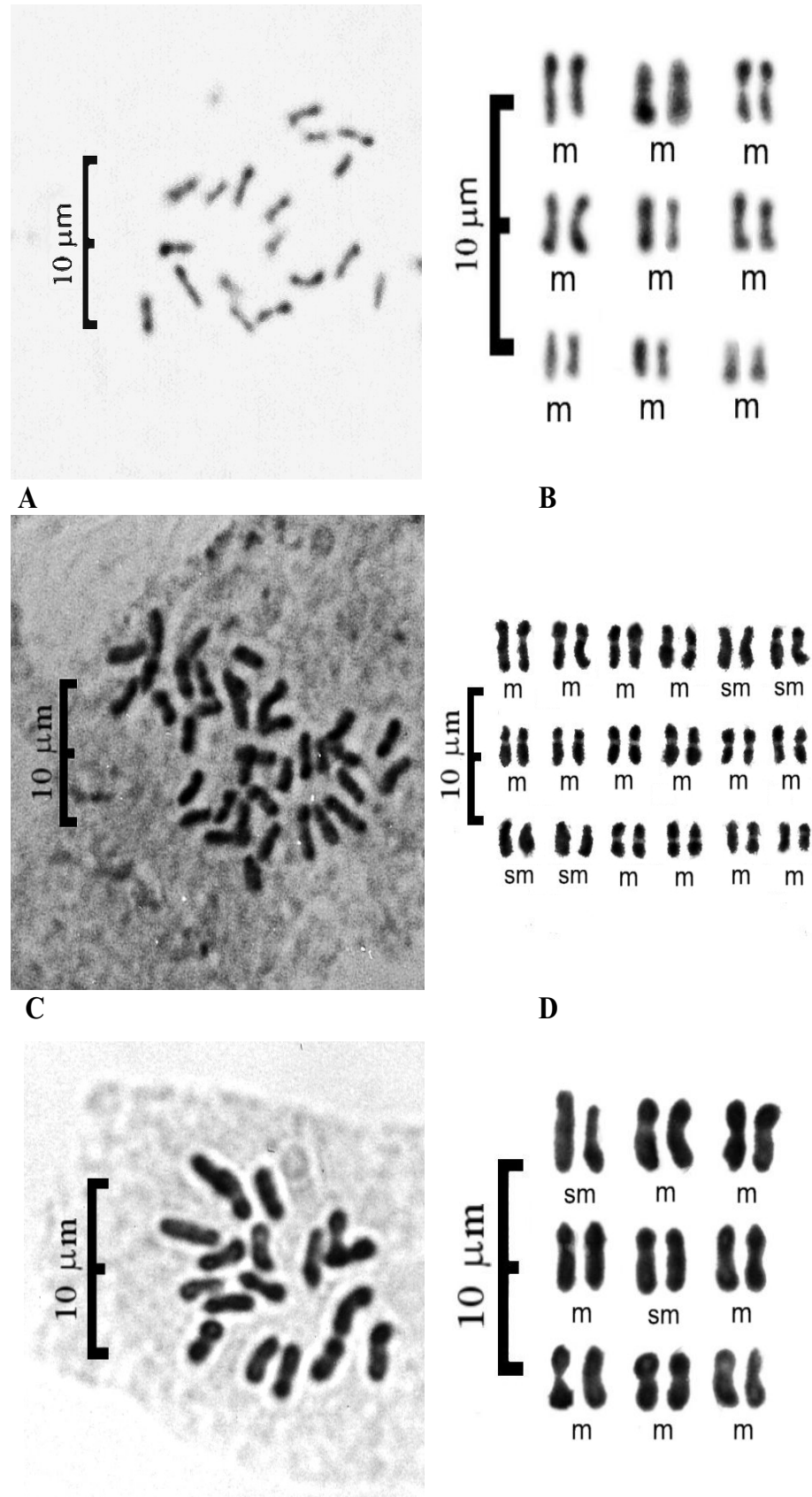

D

$\mathbf{E}$

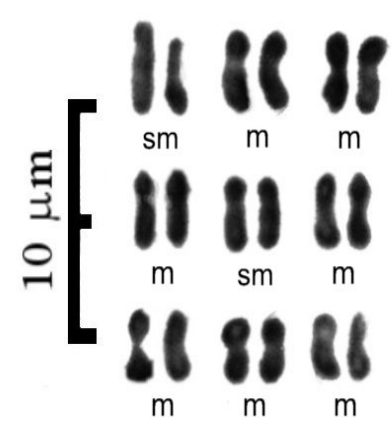

$\mathbf{F}$

Figura 4. Metafases somáticas y cariotipos de: A-B, C. langeana subsp. langeana (Salamanca, COFC 30713); C-D, C. aristata (Madrid, COFC 30686); E-F, C. castellanoides subsp. arundana (Málaga, UNEX 34477). 
Baterno, camino a La Garganta, 10-VII-2005, E. López (UNEX 34474). Ciudad Real. Luciana, carretera a Puebla de Don Rodrigo 3-VII-2004, E. López (COFC 30716).

Se han estudiado dos poblaciones, encontrándose en las plantas de ambas el mismo número cromosómico, $2 n=18$ (fig. 5 , E-F). La asimetría del cariotipo es de tipo A1, las fórmulas cromosómicas encontradas son $6 \mathrm{~m}+3 \mathrm{sm}$ (UNEX 34474) y $8 \mathrm{~m}+1 \mathrm{sm}$ (COFC 30716), y el tamaño de los cromosomas muy similar: $3,3 \mathrm{~mm}-(2,48$ $\mathrm{mm} \pm 0,36)-2,12 \mathrm{~m}(\mathrm{UNEX} 34474)$ y $3,1 \mathrm{~mm}-$ $(2,78 \mathrm{~mm} \pm 0,24)-2,3 \mathrm{~mm}($ COFC 30716$) . \mathrm{Se}$ trata, probablemente, de los primeros datos cariológicos para este endemismo del C-SW de la Península Ibérica (López \& Devesa, 2008b).

18. C. schousboei Lange in Vidensk. Meddel. Dansk Naturhist. Foren. Kjøbenhavn 1861(1-7): 135 (1862)

Material estudiado. ESPAÑA. Badajoz. Monesterio, sierra de La Culebrera, 20-VI-2004, Valtueña \& Relinque (COFC 30738).

En plantas de la única población estudiada se ha encontrado el número $2 n=36$ (fig. $6, \mathrm{~A}-\mathrm{B}$ ), que indica su origen poliploide $(4 x, x=9)$. La asimetría cariotípica es tipo A1 y la fórmula cromosómica $16 \mathrm{~m}+2 \mathrm{sm}$; el tamaño de los cromosomas viene definido por los valores 3,0 $\mathrm{mm}-(2,12 \mathrm{~mm} \pm 0,38)-1,9 \mathrm{~mm}$. Se trata del primer recuento realizado en este taxon, ya que el efectuado por Fernández Casas \& Gamarra (1986) en una supuesta población leonesa, corresponde en realidad a C. langeana Willk.

\section{CENTAUREA GR. C. ALBA L.}

19. C. alba L. Sp. Pl.: 914 (1753) subsp. alba var. alba

Material estudiado. España. Madrid. Entre Los Molinos y Guadarrama, 7-VII-2005, E. López (UNEX 34484). Valladolid. Entre Ataquines y Olmedo, 2-VII-2003, Ortega \& E. López (COFC 30407).

En plantas de las dos poblaciones estudiadas se ha encontrado el mismo número cromosómico, $2 n=18$ (fig. 6, C-D), que coincide (tab. 2) con los hallados por Gardou (1972a) y Hellwig (1994) con material procedente de Madrid y otras localidades españolas, y por Agudo (1981) con material de Ávila (sub C. alba subsp. macrocephala Pau). Los cariotipos estudiados presentan asimetría de tipo A1, las fórmulas cromosómicas $5 \mathrm{~m}+1 \mathrm{~m}-\mathrm{sm}+3 \mathrm{sm}$ (UNEX 34484) y $8 \mathrm{~m}+1 \mathrm{sm}$ (COFC 30407$)$, y un tamaño de los cromosomas comprendido entre $3,5 \mathrm{~mm}$ $(2,93 \mathrm{~mm} \pm 0,36)-2,5 \mathrm{~mm}($ UNEX 34484) y 3 $\mathrm{mm}-\left(2,48^{\circ} \mathrm{mm} \pm 0,32\right)-2,1 \mathrm{~mm}($ COFC 30407).

20. C. alba subsp. tartesiana Talavera in Lagascalia 12: 248 (1984)

Material estudiado. ESPAÑA. Huelva: entre Aguafría y El Quejigo, 22-VII-2005, E. López (UNEX 34488).

En plantas de la población estudiada se ha encontrado el número $2 n=18$ (fig. 6, E-F), que coincide con el único recuento previo de que se tiene constancia (Pastor et al., 1990). La fórmula cromosómica hallada es $3 \mathrm{~m}+2 \mathrm{~m}^{\text {sat }}+4 \mathrm{sm}$, la asimetría cariotípica de tipo A1 y el tamaño de los cromosomas encontrado viene definido por los valores $3,5 \mathrm{~mm}-(2,39 \mathrm{~mm} \pm 0,55)-1,8 \mathrm{~mm}$.

21. C. costae Willk. in Linnaea 30: 115 (1859) var. costae

Material estudiado. ESPAÑA. Huesca. Jaca, camino al parador de Oroel, 20-VII-2004, J. López \& E. López (UNEX 34487).

El número cromosómico encontrado, $2 n=$ 18 (fig.7, A-B) es, probablemente, el primer recuento efectuado para este endemismo del NE de España. La fórmula cromosómica hallada es $7 \mathrm{~m}+2 \mathrm{sm}$, la asimetría cariotípica de tipo A1 y el tamaño de los cromosomas viene definido por los valores $2,1 \mathrm{~mm}-(1,68 \mathrm{~mm} \pm 0,29)-1,3 \mathrm{~mm}$.

22. C. costae var. maluqueri Font Quer in Treb. Mus. Ci. Nat. Barcelona 5: 230 (1920) Material estudiado. ESPAÑA. Lérida. Pobla de Segur, 21-VII-2004, J. López \& E. López (UNEX 34486).

Se ha encontrado el número $2 n=18$ (Lámina 7, C-D) en plantas procedentes de la localidad típica de este endemismo del NE de España, que al parecer se estudia por vez primera, y que coincide con el hallado en el resto de los táxones de la sección. La fórmula cromosómica es $7 \mathrm{~m}+$ $2 \mathrm{sm}$, la asimetría cariotípica de tipo A1 y el tamaño de los cromosomas viene definido por los 


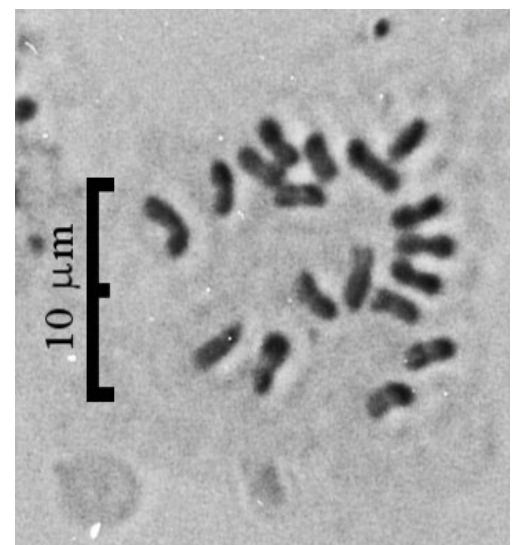

A

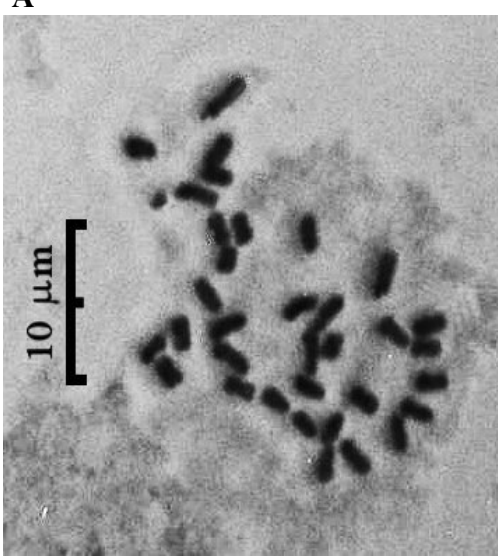

C

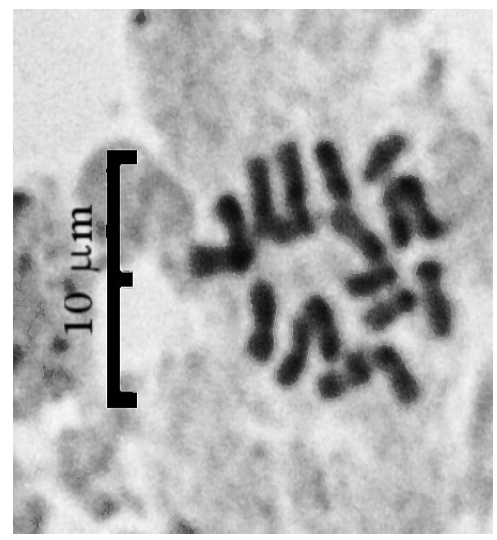

E

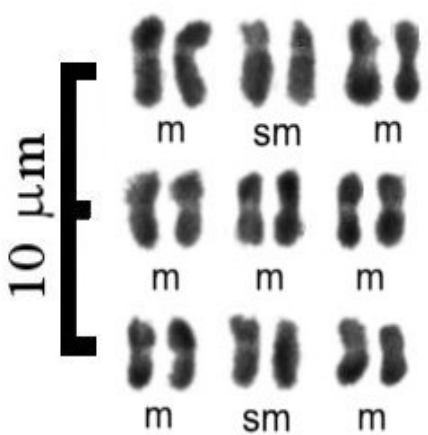

B

D

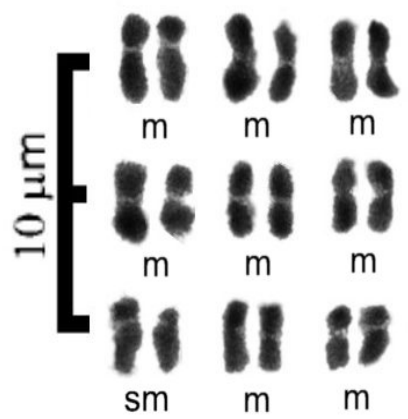

$\mathbf{F}$

Figura 5. Metafases somáticas y cariotipos de: A-B, C. castellanoides subsp. talaverae (Albacete, UNEX 35212); C-D, C. cordubensis (Huelva, UNEX 35206); E-F, C. bethurica (Ciudad Real, COFC 30716). 
valores $2,1 \mathrm{~mm}-(1,61 \mathrm{~mm} \pm 0,25)-1,3 \mathrm{~mm}$.

\section{DISCUSIÓN}

Los recuentos cromosómicos efectuados en táxones del grupo de $C$. paniculata y en el de $C$. alba indican el predominio del número cromosómico $2 n=18$, como era de esperar de acuerdo con los numerosos antecedentes previos (tabs. 1 y 2), habiéndose detectado la presencia de cromosomas accesorios tan solo en un población de $C$. hanryi $(2 n=18+1 \mathrm{~B})$, casuística de la que también existen antecedentes en algunos táxones. El número básico $x=9$, que caracteriza ambos grupos, es también el del grupo Willkommia (Blanca, 1980 \& 1981c), íntimamente relacionado de acuerdo con las filogenias moleculares (Font et al., 2002; Garcia-Jacas et al., 2006; Suárez-Santiago et al., 2007).

El predominio de los citótipos diploides $(2 n=18, x=9)$ constituye la generalidad, $\mathrm{y}$ su detección en muchos de los táxones constituye una novedad, al haber sido estudiados por vez primera desde este punto de vista, como sucede en $C$. paniculata subsp. exilis, C. paniculata subsp. geresensis, C. coutinhoi, C. kheilii, $C$. limbata var. insularis, C. castellanoides subsp. talaverae, C. cordubensis, $C$. bethurica y $C$. schousboei, del grupo $C$. paniculata, y en $C$. costae var. costae y $C$. costae var. maluqueri, del grupo C. alba. Por el contrario, la incidencia de la poliploidía es escasa, pero sin duda está en el origen al menos de C. aristata, C. cordubensis y $C$. schousboei, para las que se ha encontrado el nivel tetraploide $(2 n=36,4 x)$, un fenómeno que ya había sido señalado previamente tanto para el grupo de $C$. paniculata [también en C. aristata, Agudo (1981, sub C. castellana)] como de C. alba [C. alba, Kuzmanov, Georgieva et al. (1986); C. alba subsp. subcialiaris, Phitos \& Damboldt (1971) y $C$. huljakii, Matthäs (1976)].

En el caso de $C$. cordubensis $(2 n=36$, $4 x$ ) la condición poliploide podría haber favorecido la dominancia de estas plantas en ambientes más xéricos, toda vez que la muy afín $C$. bethurica $(2 n=18,2 x)$ encuentra más favorables los ambientes montanos menos térmicos y más húmedos (López \& Devesa, 2008a). El carácter tetraploide de $C$. schousboei $(2 n=36,4 x)$, especie con algunas características intermedias entre $C$. cordubensis $(2 n=36)$ y $C$. alba subsp. tartesiana $(2 n=18)$, y su notable frecuencia en el SW de España, sugiere que se trata de una microespecie estabilizada, con un areal definido entre los posibles parentales de origen, descartándose que se trate de un híbrido ocasional entre ambos táxones (López \& Devesa, 2008a), como habían sugerido algunos autores (Pau, 1928). Para explicar su origen y la retención de caracteres intermedios entre los táxones citados, al menos teóricamente, puede recurrirse a fenómenos de alopoliploidía entre el antecesor diploide $2 n=18$ de $C$. cordubensis (o la misma $C$. bethurica) y $C$. alba subsp. tartesiana $(2 n=18)$, cuyo anfidiploide $2 n=$ 18 daría lugar por autopoliplodía al tetraploide $2 n=36$, con características intermedias.

En todos los casos estudiados de ambos grupos, el tamaño de los cromosomas es similar, pudiéndose tipificar de pequeños o medianamente pequeños -medianamente grandes en algunos táxones del grupo de $C$. paniculata- de acuerdo con la terminología de Stebbins (1971), oscilando su tamaño entre 1,2 y $5,2 \mathrm{~mm}$ en el grupo de $C$. paniculata, y entre 1,2 y $3,5 \mathrm{~mm}$ en el de $C$. alba, con un neto predominio de cromosomas metacéntricos $\mathrm{y}$, en menor medida, submetacéntricos.

Finalmente, respecto de la asimetría cariotípica hallada señalar que, con las limitaciones que impone la dificultad de 

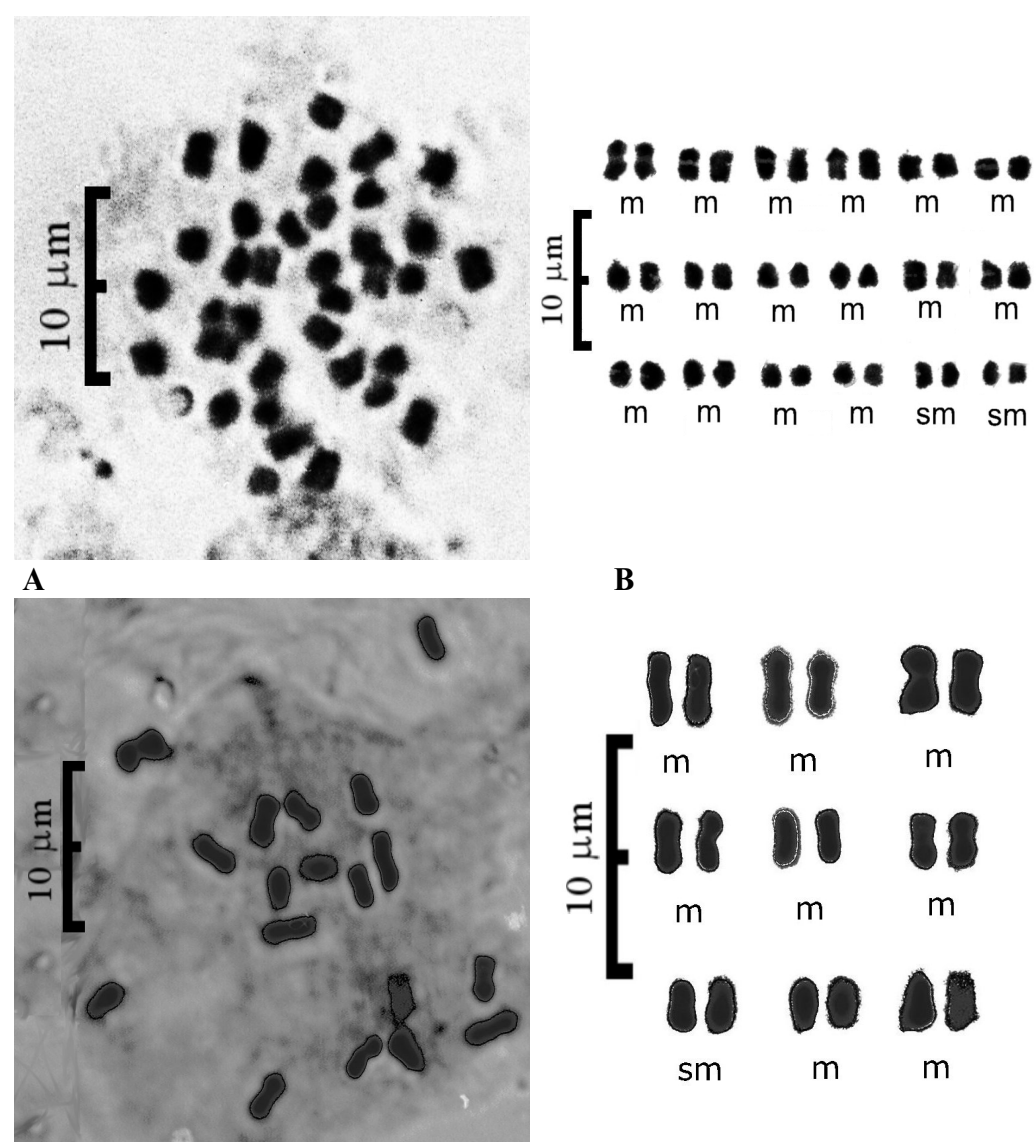

B
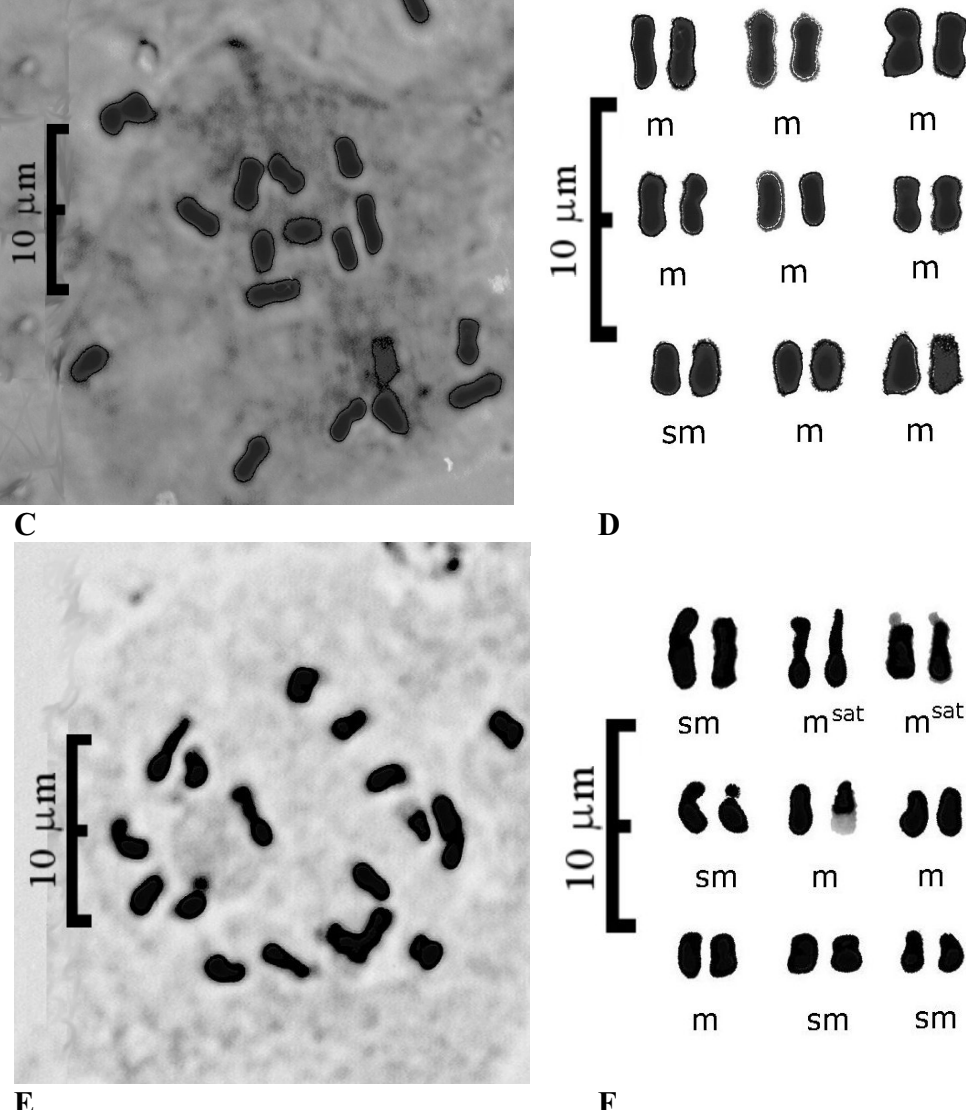

D

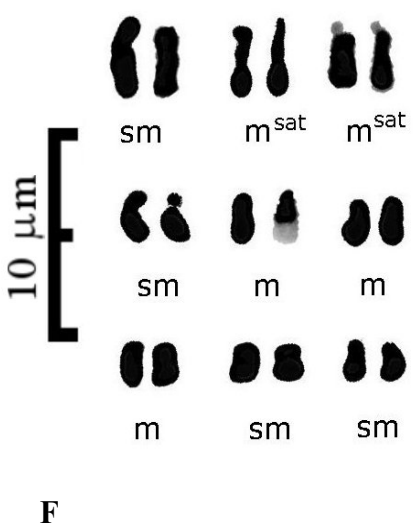

Figura 6. Metafases somáticas y cariotipos de: A-B, C. schousboei (Badajoz, COFC 30738); C-D, C. alba subsp. alba var. alba (Valladolid, COFC 30407); E-F, C. alba subsp. tartesiana (Huelva, UNEX 34488). 


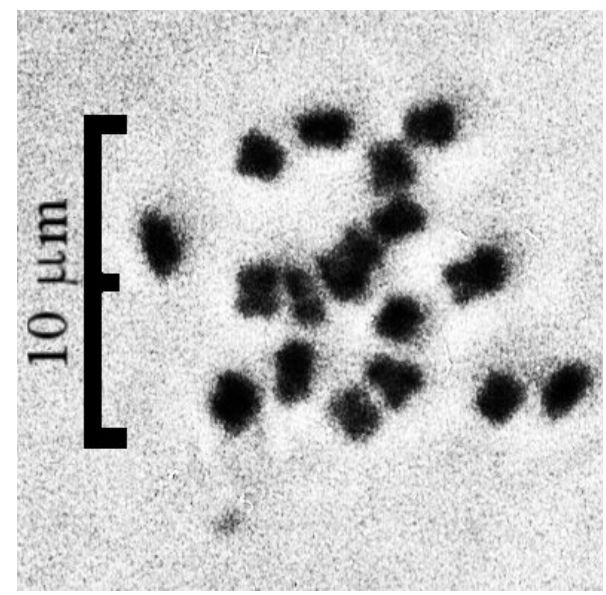

A

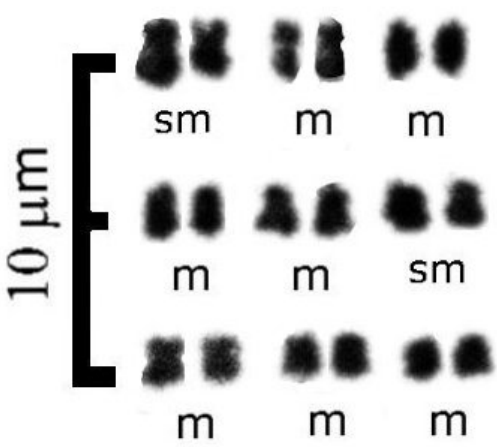

B
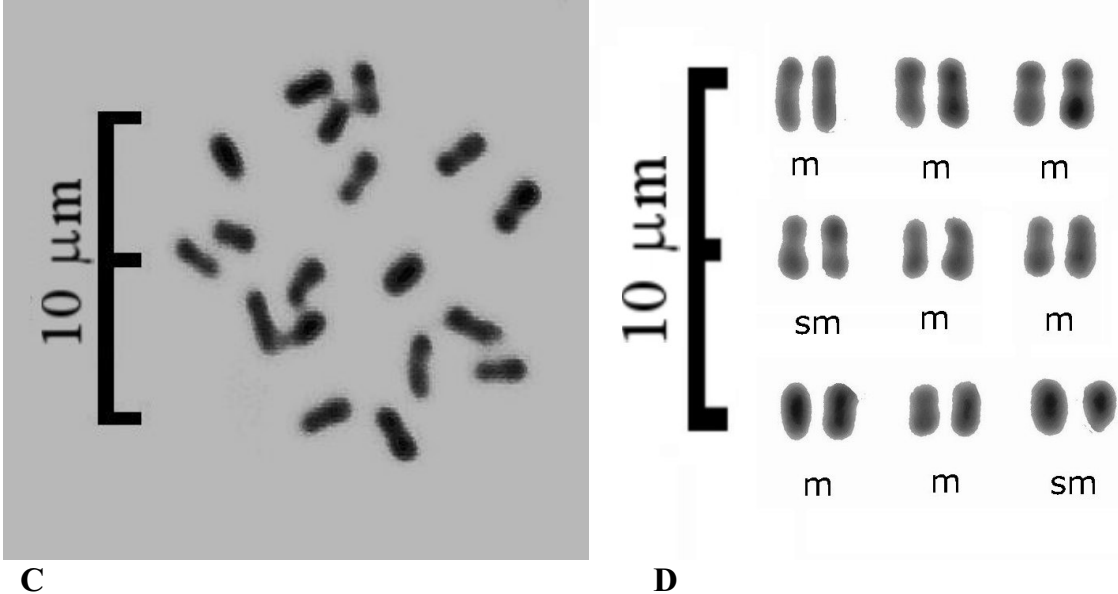

Figura 7. Metafases somáticas y cariotipos de: A-B, C. costae var. costae (Huesca, UNEX 34487); CD, C. costae var. maluqueri (Lérida, UNEX 34486).

estudio del material y la poca definición de los cromosomas, en todas las poblaciones estudiadas es de tipo A1, excepto en el caso de la población COFC 30692 de C. langeana subsp. exilis, que es B1. Presentan, pues, un cariotipo con alto grado de simetría, lo que sugeriría que se trata de táxones poco especializados siguiendo las ideas de Stebbins (1971), lo que no parece estar en consonancia con las características del grupo de acuerdo con los datos de filogenia, lo que sugiere que mecanismos como las traslocaciones, ya conocidos en el género (Garcia-Jacas et al., 1996), han debido contribuir en este grupo al aumento de simetría.

AGRADECIMIENTOS. El presente trabajo ha sido desarrollado en el contexto de la obra Flora Iberica, y financiado por los proyectos REN200204634-C05-04 y CGL2005-05471-C04-02, de la Dirección General de Investigación Científica y 
Técnica del Ministerio de Educación y Ciencia. Uno de los autores, E. López, estuvo adscrito como becario en formación (BES-2003-1275) a cargo de los proyectos citados.

\section{BIBLIOGRAFÍA}

AFZELIUS, K. -1924- Embriologische und zytologische Studien in Senecio und verwandten Gattungen. Svensk Bot. Tidskr. 16: 371-382.

AGUDO, M. P. -1981- Aportación al estudio citotaxonómico del género Centaurea L. (Compositae) en la Península Ibérica. Tesis Doctoral. Universidad Complutense de Madrid. Madrid.

APARICIO, A. -1993- Planes de recuperación de especies vegetales amenazadas en el Parque Natural de la Sierra de Grazalema (CádizMalaga). Acta Bot. Malacitana 18: 199-221.

ARÈNES, J. -1949- Les races lusitaniennes de Centaurea paniculata L. sensu latísimo. Agron. Lusit. 11: 5-32.

ARÈNES, J. -1951- Le groupe spécifique du Centaurea paniculata L. sensu latiss. Mém. Mus. Natl. Hist. Nat. Paris, Sér. B, Bot. 1: 175-266.

ARRIGONI, P.V. \& B. MORI -1971- Numeri cromosomici per la flora italiana: 92-97. Inform. Bot. Ital. 3: 226-233.

BLANCA, G. -1980- Notas cariosistemáticas en el género Centaurea L. Sect. Acrocentroides Willk. I. Anales Jard. Bot. Madrid 36: 349369.

BLANCA, G. -1981a- Revisión del genero Centaurea L. Sect. Willkommia G. Blanca, nom. nov. Lagascalia 10: 131-205.

BLANCA, G. -1981b- Estudios taxonómicos en el género Centaurea L. Sección Willkommia G. Blanca: Palinología. Bot. Macaron. 8-9: 103-118.

BLANCA, G. -1981c- Notas cariosistemáticas en el género Centaurea L. sect. Willkommia G. Blanca. II. Conclusiones. Anales Jard. Bot. Madrid 38: 109-125.

BRAMWELL, D., C. J. HUMPHRIES \& B. G. MURRAY -1972-Chromosome studies in the flora of Macaronesia. Bot. Not. 125: 139-152. BRAMWELL, D., C. J. HUMPHRIES, B. G.
MURRAY \& J. OWENS - 1971- Chromosome numbers in plants from the Canary islands. Bot. Not. 124: 376-382.

BRULLO, S., A. GUGLIELMO, P. PAVONE \& M. C. TERRASI-1991- Numeri cromosomici per la flora Italiana: 1251-1266. Inform. Bot. Ital. 23: 39-46.

CAIXINHAS, L., T. VASCONCELOS, J. MONJARDINO, A. DUARTE BARÃO, C. PINTO RICARDO \& J. NEVES MARTINS 1991- Situation actuelle de la banque de germoplasme des espèces endémiques du Portugal. Bot. Chron. (Patras) 10: 603-608.

CONSTANTINIDIS, T. \& G. KAMARI -1995Mediterranean chromosome number reports 5 (401-414). Fl. Medit. 5: 265-278.

CONSTANTINIDIS, T. \& G. KAMARI -2000- A karyological study of ten taxa of phanerogams (Compositae, Leguminosae, and Umbelliferae) from Greece. Bot. Chron.(Patras) 13: 117-131.

DAMBOLDT, J., G. GRAUMANN \& U. MATTHÄS -1973- IOPB Chromosome number reports, XXXIX. In: Á. LövE - ed.- . Taxon 22: 116.

DAMBOLDT, J. \& U. MATTHÄS -1975Chromosomezahlen einiger mediterraner und mitteeleuropäischer Centaurea-Arten (Asteraceae). Pl. Syst. Evol. 123: 107-115.

DAMBOLDT, J. \& U. MATTHÄS -1979Karyologische Untersuchungen an Centaurea (sect. Phalolepis) pawlowskii (Compositae). Bot. Jahrb. Syst. 100: 406-413.

DAMBOLDT, J \& V. MELZHEIMER -1974Über einige Centaurea-Arten (Asteraceae) der Athos-Halbinsel. Bot. Jahrb. Syst. 94: 383394.

DELAY, J. -1969- Halophytes III. Inf. Ann. Caryosyst. Cytogenet. 3: 17-27.

DIPALI DEY, A.K.S. -1967- Chromosome studies in the genus Centaurea. Folia Biol. (Cracow) 15: 191-207.

DOSTÁL, J. -1976-Centaurea L. In: T.G. TUTIN, V.H. HEYWOOD, N.A. BURGES, D. H. VALENTINE, S. M. WALTERS \& D. A. WEBB -eds.- Flora Europaea 4: 254-301. Cambridge University Press. Cambridge.

DVORAK, K., B. DADAKOVA \& I. RUZICKA -1979- Chromosome Morphology of the Czechoslovak of the Genus Scorzonera. Folia Geobot. Phytotax. Bohemoslov. 14: 185-199. 
FEDOROV, A. A. -1974- Chromosome numbers of flowering plants. Otto Koeltz, Koenigstein FERNANDES, A \& M. QUEIRÓS -1971Contribution á la connaissance cytotaxonomique des. Spermatophyta du Portugal. II. Compositae. Bol. Soc. Brot., Ser. 2, 45: 5-121.

FERNÁNDEZ CASAS, F. J. \& M. J. FERNÁNDEZ MORALES -1979- Centaurea lainzii, un triploide natural. Mém. Soc. Bot. Genève 1: 115-122.

FERNÁNDEZ CASAS, J. \& R. GAMARRA 1986- IOPB Chromosome number reports XCI. In: Á. Lóve -ed.-. Taxon 35: 406.

FERNÁNDEZ CASAS, F. J. \& A. SUSANNA 1986- Monografía de la sección Chamaecyanus Willk. del género Centaurea L. Treb. Inst. Bot. Barcelona 10.

FERNANDEZ MORALES, M. J. -1974- Estudios citogenéticos en especies españolas del género Centaurea, sec. AGUDO (1981).

FERNÁNDEZ MORALES, M. J. \& C. GARDOU -1975- Caryosystematic studies of some species of the genus Centaurea in the Western Mediterranean Basin. In: S. M. WALTERS -ed.European Floristic and Taxonomic Studies: 61-75. Conference Report (Botanical Society of the British Isles).

FONT, M., T. GARNATJE, N. GARCIA-JACAS \& A. SUSANNA -2002- Delineation and phylogeny of Centaurea sect. Acrocentron based on DNA sequences: a restoration of the genus Crocodylium and indirect evidence of introgression. Pl. Syst. Evol. 234: 15-26.

GABRIELIAN, E. T. -1995- On the generic status of certain groups of Centaureinae (Compositae). In: D. J. N. HIND, C. JEFFREY \& G. V. POPE -eds.- Advances in Compositae Systematics:145-152. Royal Botanic Garden. Kew.

GARCIA-JACAS, N. -1998- Centaurea kunkelii (Asteraceae, Cardueae), a new hybridogenic endecaploid species of sect. Acrocentron from Spain. Ann. Bot. Fenn. 35: 159-167.

GARCIA-JACAS, N. \& A. SUSANNA -1992Karyological notes on Centaurea sect. Acrocentron. Pl. Syst. Evol. 179: 1-18.

GARCIA-JACAS, N., A. SUSANNA, T. GARNATJE \& R. VILATERSANA -2001Generic Delimitation and Phylogeny of the
Subtribe Centaureinae (Asteraceae): A Combined Nuclear and Chloroplast DNA Analysis. Ann. Bot. (Oxford) 87: 503-515.

GARCIA-JACAS, N., A. SUSANNA \& R. ILARSLAN -1996- Aneuploidy inCentaureinae (Compositae): is $\mathrm{n}=7$ the end of the series?. Taxon 45: 39-42.

GARCIA-JACAS, N., A. SUSANNA, R. ILARSLAN \& H. ILARSLAN -1997- New chromosome counts in the subtribe Centaureinae (Asteraceae, Cardueae) from West Asia. Bot. J. Linn. Soc. 125: 343-349.

GARCIA-JACAS, N., A. SUSANNA \& V. MOZAFFARIAN -1998b- New chromosome counts in the subtribe'Centaureinae (Asteraceae, Cardueae) from West Asia, III. Bot. J. Linn. Soc. '128: 413-422.

GARCIA-JACAS, N., A. SUSANNA, V. MOZAFFARIAN \& R. ILARSAN -2000- The natural delimitation of Centaurea (Asteraceae: Cardueae): ITS sequence analysis of the Centaurea jacea group. $\mathrm{Pl}$. Syst. Evol. 223: 185-199.

GARCIA-JACAS, N., A. SUSANNA, R. VILATERSANA \& M. GUARA -1998a- New chromosome counts in the subtribe Centaureinae (Asteraceae, Cardueae) from West Asia, II. Bot. J. Linn. Soc. '128: 403-412.

GARCIA-JACAS, N., T. UYSAL, K. ROMASCHENKO, V. N. SUÁREZ SANTIAGO, K. ERTUGRUL \& A. SUSANNA -2006- Centaurea Revisited: A Molecular Survey of the Jacea Group. Ann. Bot. 98: 741-753

GARCÍA MARTÍNEZ, X. R. -2000- Números cromosomáticos de plantas occidentales, 818826. Anales Jard. Bot. Madrid 58: 163.

GARDOU, C. -1969- Caryosystématique des Centaurées de la section Acrocentron Cass. Bull. Soc. Bot. France 116: 29-38.

GARDOU, C. -1972a- IOPB Chromosome numbers reports XXXVII. In: Á. LövE -ed.Taxon 21: 495-500.

GARDOU, C. -1972b- Recherches biosystématiques sur la Section Jacea Cass. et quelques sections voisines du genre Centaurea L. en France et dans les régions limitrophes. Feddes Repert. 85: 311-472.

GEORGIADIS, T. \& D. PHITOS -1976Contribution à l'étude cytotaxonomique du 
genre Centaurea L. (sectio Acrolophus (Cass.) DC.) en Grèce. Biol. Écol. Médit. 3: 13-16. GREUTER, W., G. WAGENITZ, M. AGABABIAN \& H. HELLWIG -2001Proposal to conserve the name Centaurea (Compositae) with a conserved type. Taxon 50: 1201-1205.

GUINOCHET, M. -1956- Sur l'existence d'une forme systématique normalement trisomique chez Centaurea paniculata L. C. R. Compt. Rend. Acad. Sci. Paris, Sér. 3, Sci. Vie 243: 2130-2132 (sec. FEDOROV, 1974).

GUINOCHET, M. -1957- Contribution à l'etude caryologique du genre Centaurea L. sens lat. Bull. Soc. Hist. Nat. Afrique N. 48: 282-300.

GUINOCHET, M \& J. FOISSAC -1962- Sur les caryotypes de quelques espèces du genre Centaurea L. et leur signification taxonomique. Rev. Cytol. Biol. Veg. 25: 373-389.

HELLWIG, H. F. -1994- Chromosomenzahlen aus der Tribus Cardueae (Compositae). Willdenowia 24: 219-248.

JEFFREY, C. (2007) Compositae. In: J.W. KADEREIT \& C. JEFFREY eds.-The families and genera of vascular plants, 61-87. Springer.

KAMARI, G. -1996- In: T. CONSTANTINIDIS \& D. VASSILIADES, Centaurea musarum (Compositae): the rediscovery of a rare endemic species. Bot. Chron. (Patras) 12: 914.

KIEHN, M., E. VITEK \& C. DOBE $\square$ A -2000In: C. DOBE $\square \mathrm{A} \&$ E. VITEK -eds.Documented Chromosome Number Checklist of Austrian Vascular Plants. Naturhistorischen Museums Wien, Viena (sec. Index to Plant Chromosome numbers, IPCN, http://mobot.mobot.org/W3T/Search/ ipen.html).

KUZMANOV, B. A., S. B. GEORGIEVA \& V. A. NIKOLOVA-1986-Chromosome numbers of Bulgarian flowering Plants. Fitologija 31: 71-74.

LEVAN, A., K. FREDGA \& A. A. SANDBERG -1964- Nomenclature for centromeric position on chromosomes. Hereditas 52: 201220.

LÓPEZ, E. -2008- Estudio taxonómico de Centaurea sect. Paniculatae (Hayek)Dostál y sect. Phalolepis (Cass.) DC. en la Península Ibérica. Tesis Doctoral. Universidad de
Extremadura. Badajoz.

LÓPEZ, E. \& J. A. DEVESA. -2008a- Notas taxonómicas sobre el género Centaurea L. (Asteraceae) en la Península Ibérica. I. C. cordubensis Font Quer, C. bethurica E. López \& Devesa, sp. nova. y C. schousboei Lange. Anales Jard. Bot. Madrid 65: 331-342.

LÓPEZ, E. \& J. A. DEVESA. -2008b- Notas taxonómicas sobre el género Centaurea L. (Asteraceae) en la Península Ibérica. II. Centaurea castellanoides Talavera y C. aristata Hoffmanns. \& Link. Acta Bot Malacitana 33: 57-68.

MARDSEN-JONES, E. M. \& W. B. TURRILL 1937- Genetical studies in Centaurea scabiosa L. and Centaurea collina L. J. Genet. 34: 487495.

MATTHÄS, U. -1976- Zur Cytotaxonomie von Centaurea subciliaris Boiss. \& Heldr. (Sektion Phalolepis (Cass.) DC.) und verwandter Sippen im europaischen Mediterrangebiet. I. Bot. Jahrb. Syst. 95: 418-434.

MORINAGA, T., T. KANO, Y. MARUYANA \& Y. YAMASAKI -1929- Chromosome numbers of cultivated plants II. Bot. Mag. (Tokyo) 43: 589-594.

NATARAJAN, G. -1981- IOPB Chromosome number reports LXXII. In: Á. LÖVE ed. Taxon 30: 698-699.

NATARAJAN, G. -1988- Étude caryosystématique de quelques dicotyledones de la Garrigue Languedocienne. Naturalia Monspel., Sér. Bot. 52: 85-123.

NILSSON, Ö. \& P. LASSEN -1971Chromosome numbers of vascular plants from Austria, Mallorca and Yugoslavia. Bot. Not. 124: $270-276$.

OCHSMANN, J. -1999- Chromosomenzahlen einiger europäischer Centaurea-Sippen. Haussknechtia 7: 59-65.

OCHSMANN, J. -2000- Morphologische und molekularsystematische Untersuchungen an der Centaurea stoebe L.-Gruppe (AsteraceaeCardueae) in Europa. Diss. Bot. 324.

PASTOR, J., J. C. DIOSDADO, C. SANTA BÁRBARA, J. VIOQUE \& E. PÉREZ -1990Números cromosómicos para la flora española, 556-591. Lagascalia 15: 269-282.

PAU, C. (1928) Notas de mi herbario. Cavanillesia 1: 60-67. 
PHITOS, D. -1970- Zur polyploidie in der Gattung Centaurea L. Sektion Acrocentron. Ber. Deutsch. Bot. Ges. 83: 69-74.

PHITOS, D. -1971- Cytotaxonomische studien des griechischen Centaurea-Arten Sektion Acrocentron (Compositae). Ber. Deutsch. Bot. Ges. 84: 255-259.

PHITOS, D. \& J. DAMBOLDT -1971- Beiträge zur Flora Ionica. III. Cytotaxonomische Bemerkungen zu einigen griechischen Compositen. Ann. Naturhist. Mus. Wien 75: 157-162.

PLITMANN, U. -1976- Taxonomic studies in Centaurea sect. Calcitrapa. III. Cytotaxonomic notes. Israel J. Bot. 25: 84-89.

PODDUBNAJA-ARNOLDI, V. -1927Spermatogenesis bei einigen Compositen. Planta 7: 284-298.

PODDUBNAJA-ARNOLDI, V. -1931- Ein Versuch der Anwendung der embryologischen Methode bei der Lösung einiger Systematischen Fragen I. Vergleichend embryologisch-zytologische Untersuchungen über die Gruppe Cynareae, Fam. Compositae. Beih. Bot. Centralbl. II, 48: 141-237.

QUEIRÓS, M. -1973- Contribuição para o conhecimiento citotaxonomico das Spermatophyta de Portugal. II. Compositae. Bol. Soc. Brot., 47 Supl.: 299-314.

ROY, B. -1938- Chromosome numbers in some species and hybrids of Centaurea. J. Genet. 35: 90-95.

RUNEMARK, H. -1967- Studies in the Aegean flora. XII. Cytologic and morphologic investigations in Centaurea. Bot. Not. 120: 161-176.

SILJAK-YAKOVLEV, S. (1977) IOPB chromosome number reports LVII. In Á. LövE ed. Taxon 26: 443-452.

SILJAK-YAKOVLEV, S. (1986) Etude cytogénétique el palynologique de Compositae endémiques ou reliques de la flore yougoslave. Thesis, University of Paris-Sud, Paris.

SNOW, R. -1963- Alcoholic hydrochloric acidcarmine as a stain for chromosomes in squash preparations. Stain Technol. 38: 9-13.

SOLBRIG, O. T. -1977- Chromosome cytology and evolution in the family Compositae. In: V. H. HEYWOOD, J. B. HARBORNE \& B. L. TURNER (eds.) The biology and the chemistry of the Compositae: 246-260. Academic Press. London \& New York.

SPETA, F. -1971- Chromosomenzahlen einiger Angiospermen. Oesterr. Bot. Z. 119: 1-5.

STEBBINS, G. L. -1950-Variation and Evolution in Plants. Columbia, New York.

STEBBINS, G. L. -1971- Chromosomal evolution in higher plants. Edward Arnold, London.

SUAREZ SANTIAGO, V. N., M. J. SALINAS, N. GARCIA-JACAS, P. S. SOLTIS, D. E. SOLTIS \& G. BLANCA -2007- Reticulate evolution in the Acrolophus subgroup (Centaurea L., Compositae) from the western Mediterranean: origin and diversification of section Willkommia Blanca. Mol. Phyl. Evol. 43: 156-172.

TANAKA, N. Y. -1981- Studies on chromosome arrangement in some higher plants III. Haplopappus gracilis $(2 n=4)$ and Crepis capillaris $(2 \mathrm{n}=6)$. Cytologia 46 : 545-559.

TJIO, JH. \& A. LEVAN -1950- The use of oxyquinoline in chromosome analysis. Anales Estac. Exp. Aula Dei 2: 21-64.

TONIAN, Z. R. -1968- The chromosome numbers of some species of the genus Centaurea L. Biol. Zhurn. Armenii 21: 86-96.

VALDÉS-BERMEJO, E. \& J. GÓMEZ GARCÍA -1976- Notas cariosistemáticas sobre flora española. I. Acta Bot. Malacitana 2: 39-50.

VAN LOON, J. C. \& H. DE JONG -1978- IOPB Chromosome number reports LIX. In: Á. LÖVE -ed.- Taxon 27: 56-60.

VAN LOON, J.C. \& B. KIEFT (1980) IOPB Chromosome numbers reports XXXIV. In: Á. Löve -ed.- Taxon 29: 538-542.

WAGENITZ, G. -1955- Pollenmorphologie und Systematik in der Gattung Centaurea L. s. 1. Flora 142: 213-279.

WATANABE, K., C. R. CARTER \& S. SMITHWHITE -1976 The cytology of Brachycome lineariloba. 6. A synchronous chromosome condensation and meiotic behaviour in $\mathrm{B}$. Iineariloba $(\mathrm{n}=2) \times$ B. campylocarpa $\mathrm{A}(\mathrm{n}=$ 4). Chromosoma 57: 319-331. 